\title{
A Reaction-Based River/Stream Water Quality Model: Reaction Network Decomposition and Model Application
}

\author{
Fan Zhang ${ }^{1, *}$, Gour-Tsyh Yeh ${ }^{2}$, Jack C. Parker ${ }^{3}$, Hongbo Zhang ${ }^{1}$, Xiaonan Shi $^{1}$, Cheng Wang ${ }^{4}$, \\ and Ruochuan $\mathrm{Gu}^{5}$ \\ ${ }^{1}$ Key Laboratory of Tibetan Environment Changes and Land Surface Processes, Institute of Tibetan Plateau Research, \\ Chinese Academy of Sciences, Beijing, China \\ ${ }^{2}$ Department of Civil and Environmental Engineering, University of Central Florida, Orlando, Florida, USA \\ ${ }^{3}$ Department of Civil and Environmental Engineering, University of Tennessee, Knoxville, Tennessee, USA \\ ${ }^{4}$ College of Water Sciences, Beijing Normal University, Beijing, China \\ ${ }^{5}$ Department of Civil and Construction Engineering, Iowa State University, Ames, Iowa, USA
}

Received 11 September 2011, accepted 23 May 2012

\begin{abstract}
This paper describes details of an automatic matrix decomposition approach for a reaction-based stream water quality model. The method yields a set of equilibrium equations, a set of kinetic-variable transport equations involving kinetic reactions only, and a set of component transport equations involving no reactions. Partial decomposition of the system of water quality constituent transport equations is performed via Gauss-Jordan column reduction of the reaction network by pivoting on equilibrium reactions to decouple equilibrium and kinetic reactions. This approach minimizes the number of partial differential advective-dispersive transport equations and enables robust numerical integration. Complete matrix decomposition by further pivoting on linearly independent kinetic reactions allows some rate equations to be formulated individually and explicitly enforces conservation of component species when component transport equations are solved. The methodology is demonstrated for a case study involving eutrophication reactions in the Des Moines River in Iowa, USA and for two hypothetical examples to illustrate the ability of the model to simulate sediment and chemical transport with both mobile and immobile water phases and with complex reaction networks involving both kinetic and equilibrium reactions.
\end{abstract}

Key words: Sediment transport, Water quality models, Rivers/streams, Chemical reactions, Chemical equilibrium/kinetics, Eutrophication Citation: Zhang, F., G. T. Yeh, J. C. Parker, H. Zhang, X. Shi, C. Wang, and R. Gu, 2012: A reaction-based river/stream water quality model: Reaction network decomposition and model application. Terr. Atmos. Ocean. Sci., 23, 605-620, doi: 10.3319/TAO.2012.05.23.02(WMH)

\section{INTRODUCTION}

As our understanding of complex biogeochemical interactions and their mathematical formulation has improved (SomlyóDy et al. 1998; Mann 2000; Yeh et al. 2001), generic transport models capable of simulating user-prescribed reaction networks have been developed which have broad applicability (Yeh and Tripathi 1989; Steefel and Cappellen 1998). A few reaction-based watershed models can handle contaminant transport with kinetic reactions (Yeh et al. 1998; Cheng et al. 2000) using a "primitive" approach, in which the partial differential equations (PDEs) governing reactive transport are integrated directly to yield distribu-

\footnotetext{
* Corresponding author

E-mail:zhangfan@itpcas.ac.cn
}

tions of water quality constituents in a region of interest over time. However, when some reactions exhibit very fast kinetics (near equilibrium behavior), the PDEs become infinitely stiff making their solution using the primitive approach intractable (Fang et al. 2003). This difficulty has been overcome by the introduction of mixed differential and algebraic equations (DAEs) for combinations of water quality constituents according to equilibrium reaction expressions and eliminating equilibrium reactions from the simultaneous solution of PDEs (Zhang et al. 2008). Most surface water DAE models require manual identification of the DAEs [DiToro 1976; Brown and Barnwell 1987 (QUAL2E); Ambrose et al. 1993 (WASP5); Cerco and Cole 1995 (CE-QUAL-ICM)]. 
Zhang et al. (2008) presented a reaction-based numerical model for sediment and reactive chemical transport in rivers and streams that utilizes a generic reaction-based approach to simulate a wide range of user-prescribed reaction processes, including diffusion, sedimentation, aqueous complexation, sorption/desorption, ion-exchange, precipitation/dissolution, reduction/oxidation, volatilization/condensation and microbially-mediated reactions (Fig. 1). Individual reactions representing any of these chemical or physical processes may be simulated as kinetic rate-limited reactions or as equilibrium reactions. Unlike most reactionbased simulators that require rate laws to be specified in a limited number of canonical forms (Fang et al. 2006), Zhang et al. (2008) describe reaction rates of elementary kinetic reactions based on collision theory (Stumm and Morgan 1981). For non-elementary kinetic reactions, the reaction rates can be formulated by user-specified rate functions based on either empirical or mechanistic approaches. Similarly, an equilibrium reaction can be described either by a thermodynamic mass action equation or by an empirical algebraic equation. Therefore, the model is able to include virtually any type of kinetic and equilibrium expressions, which makes the approach applicable to a wide range of geochemical transport problems. A matrix decomposition approach is employed to automatically determine DAEs for combinations of water quality constituents involving equilibrium and kinetic reactions.

The objective of this paper is to describe details of the matrix decomposition approach and demonstrate its application to complex reaction networks associated with sediment and dissolved phase transport in open channel flow. A field case study is presented involving eutrophication in the Des Moines River in Iowa, USA to illustrate the physical interpretation of matrix decomposition terms and to verify results by comparing simulation results with field measurements. Two hypothetical examples are presented to demonstrate the broad applicability of the model to simulate sediment and chemical transport with both mobile and immobile water phases and with complex reaction networks involving both kinetic and equilibrium reactions.

\section{MATHEMATICAL FORMULATION}

In a reaction-based formulation, $\left.\mathrm{r}_{\mathrm{i}}\right|_{\mathrm{NR}}$, the net production rate of water quality constituent $i$ due to all NR reactions $\left[\mathrm{M} \mathrm{L} \mathrm{L}^{-3} \mathrm{~T}^{-1}\right]$, is given by the summation of rates of all individual reactions in which the $\mathrm{i}$-th water quality constituent participates

$\left.\mathrm{r}_{\mathrm{i}}\right|_{\mathrm{NR}}=\sum_{\mathrm{k}=1}^{\mathrm{NR}}\left[\left(\nu_{\mathrm{ik}}-\mu_{\mathrm{ik}}\right) \mathrm{r}_{\mathrm{k}}\right]$

where $v_{\mathrm{ik}}$ is the reaction stoichiometry of the i-th water quality constituent in the $\mathrm{k}$-th reaction associated with the products, $\mu_{\mathrm{ik}}$ is the reaction stoichiometry of the i-th water quality constituent in the $\mathrm{k}$-th reaction associated with the reactants, and $r_{k}$ is the rate of the $k$-th reaction $\left[\mathrm{M} \mathrm{L}^{-3} \mathrm{~T}^{-1}\right]$.

Therefore, the temporal-spatial distribution of $\mathrm{M}$ chemical water quality constituents in a reactive transport system can be described by a set of $\mathrm{M}$ partial differential equations in the form (Zhang et al. 2008)

$$
\begin{aligned}
& \frac{\partial\left(\mathrm{A} \rho_{\mathrm{i}} \mathrm{C}_{\mathrm{i}}\right)}{\partial \mathrm{t}}+\alpha_{\mathrm{i}} \mathrm{L}\left(\rho_{\mathrm{i}} \mathrm{C}_{\mathrm{i}}\right)=\mathrm{A} \sum_{\mathrm{k}=1}^{\mathrm{N}}\left[\left(\nu_{\mathrm{ik}}-\mu_{\mathrm{ik}}\right) \mathrm{r}_{\mathrm{k}}\right], \\
& \mathrm{i} \in \mathrm{M}=\mathrm{M}_{\mathrm{im}}+\mathrm{M}_{\mathrm{m}}
\end{aligned}
$$

where $A$ is the stream cross-sectional area $\left[\mathrm{L}^{2}\right], \rho_{\mathrm{i}}$ is the density of the phase associated with water quality constituent $i$ $\left[\mathrm{M} \mathrm{L}^{-3}\right], \mathrm{C}_{\mathrm{i}}$ is the concentration of water quality constituent $\mathrm{i}\left[\mathrm{M} \mathrm{M}^{-1}\right], \mathrm{t}$ is time [T], $\alpha_{\mathrm{i}}$ is 0 for immobile water quality constituents that are not subject to longitudinal transport and 1 for mobile water quality constituents, $\mathrm{M}_{\mathrm{im}}$ is the number of immobile constituents, $\mathbf{M}_{\mathrm{m}}$ is the number of mobile constituents, $\mathrm{i} \in \mathrm{M}$ is used herein for $\mathrm{i} \in\{1, \ldots, \mathrm{M}\}$, and $\mathrm{L}$ is a transport operator including advection, dispersion, and source terms (Zhang et al. 2008).

Equation (2) can be written in matrix form as

$\mathbf{U} \frac{\partial \mathbf{C}_{\mathrm{A}}}{\partial \mathrm{t}}+\boldsymbol{\alpha L}(\mathbf{C})=\mathrm{A} \boldsymbol{v} \mathbf{r}$

where $\mathbf{U}$ is a unit matrix, for the sake of simplicity, $\mathbf{C}_{\mathbf{A}}$ is a vector with components representing $\mathrm{M}$ water quality constituent concentrations incorporating $\rho_{i}$ multiplied by the cross section area of the river, $\boldsymbol{\alpha}$ is a diagonal matrix with $\alpha_{i}$ as its diagonal component, $\mathbf{C}$ is a vector with components representing $\mathrm{M}$ water quality constituent concentrations incorporating $\rho_{\mathrm{i}}, \boldsymbol{v}$ is the reaction stoichiometry matrix, and $\mathbf{r}$ is the reaction rate vector with NR reaction rates as its components.

Equation (3) can be decomposed based on the type of reactions via the Gauss-Jordan column reduction of reaction matrix $\boldsymbol{v}$ (Chilakapati 1995). In order to avoid singularity of the reaction matrix, redundant equilibrium reactions are removed from the system prior to decomposition, so that the remaining equilibrium reactions are linearly independent (Zhang et al. 2008). Therefore, the total number of reactions $\mathrm{NR}=\mathrm{N}_{\mathrm{E}}+\mathrm{N}_{\mathrm{IK}}+\mathrm{N}_{\mathrm{DK}}$, where $\mathrm{N}_{\mathrm{E}}$ is the number of equilibrium reactions, $\mathrm{N}_{\mathrm{IK}}$ is the number of linearly independent kinetic reactions, and $\mathrm{N}_{\mathrm{DK}}$ is the number of linearly dependent kinetic reactions. Through matrix decomposition, the system of water quality constituent transport equations is transformed to a system of transport equations for linear combinations of water quality constituent concentrations. These linear combinations of constituents obtained through decomposition of the reaction matrix are defined as reaction-extents (Zhang et al. 2008). 


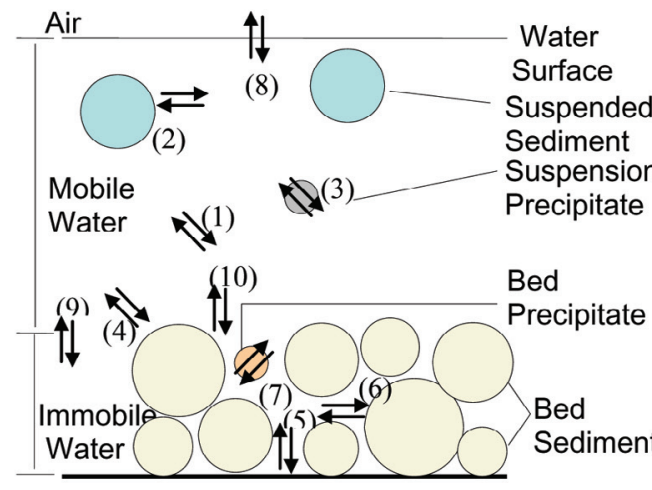

(1) Aqueous complexation in mobile water,

(2) Adsorption/desorption or ion-exchange between mobile water and suspended sediment,

(3) Precipitation/dissolution between mobile water and suspension precipitate,

(4) Adsorption/desorption or ion-exchange between mobile water and bed sediment,

(5) Aqueous complexation in immobile water,

(6) Adsorption/desorption or ion-exchange between immobile water and bed sediment,

(7) Precipitation/dissolution between immobile water and bed precipitate,

(8) Volatilization from mobile water,

(9) Diffusion between mobile and immobile water,

(10) Sedimentation of particulates between suspended and bed sediment.

Fig. 1. Ten types of reactions taken account in the model.

Through column reduction of reaction matrix $\boldsymbol{v}$ by pivoting on the $\mathrm{N}_{\mathrm{E}}$ equilibrium reactions, Eq. (3) can be incompletely decomposed into two sets. The first set contains $\mathrm{N}_{\mathrm{E}}$ equilibrium-variable transport equations involving equilibrium reactions and the second set contains $\mathrm{N}_{\mathrm{NE}}=\mathrm{M}-\mathrm{N}_{\mathrm{E}}$ nonequilibrium-variable transport equations involving no equilibrium reactions. Equilibrium-variable transport equations are then approximated with thermodynamic equilibrium equations to facilitate the decoupling of equilibrium reactions from the partial differential equations and allow robust and efficient numerical integration (Zhang et al. 2008).

Furthermore, through column reduction of reduced reaction matrix by pivoting on the $\mathrm{N}_{\mathrm{IK}}$ linear independent kinetic reactions, Eq. (3) can be completely decomposed to

$\mathbf{U}^{\prime} \frac{\partial \mathbf{C}_{\mathrm{A}}}{\partial \mathrm{t}}+\boldsymbol{\alpha}^{\prime} \mathrm{L}(\mathbf{C})=\mathrm{A}\left[\begin{array}{ccc}\mathrm{D}_{1} & \boldsymbol{v}_{1} & \boldsymbol{v}_{2} \\ \mathbf{0}_{1} & \mathrm{D}_{2} & \boldsymbol{v}_{3} \\ \mathbf{0}_{2} & \mathbf{0}_{3} & \mathbf{0}_{4}\end{array}\right]\left\{\begin{array}{l}\mathbf{r}_{\mathrm{E}} \\ \mathbf{r}_{\mathrm{IK}} \\ \mathbf{r}_{\mathrm{DK}}\end{array}\right\}$

where $\mathbf{U}^{\prime}$ is the reduced $\mathbf{U}$ matrix, $\boldsymbol{\alpha}^{\prime}$ is the reduced $\boldsymbol{\alpha}$ matrix, $\mathbf{D}_{\mathbf{1}}$ and $\mathbf{D}_{\mathbf{2}}$ are the "diagonal" submatrixes of the reduced $\boldsymbol{v}$ matrix with size of $\mathrm{N}_{\mathrm{E}} \times \mathrm{N}_{\mathrm{E}}$ and $\mathrm{N}_{\mathrm{IK}} \times \mathrm{N}_{\mathrm{IK}}$ respectively, $\boldsymbol{v}_{1}$, $\boldsymbol{v}_{2}$ and $\boldsymbol{v}_{3}$ are submatrixes of the reduced $\boldsymbol{v}$ matrix with size of $\mathrm{N}_{\mathrm{E}} \times \mathrm{N}_{\mathrm{IK}}, \mathrm{N}_{\mathrm{E}} \times \mathrm{N}_{\mathrm{DK}}$ and $\mathrm{N}_{\mathrm{IK}} \times \mathrm{N}_{\mathrm{DK}}$ separately, $\mathbf{0}_{\mathbf{1}}, \mathbf{0}_{\mathbf{2}}$, $\mathbf{0}_{3}$, and $\mathbf{0}_{4}$ are zero submatrixes of the reduced $\boldsymbol{v}$ matrix with size of $N_{I K} \times N_{E},\left(M-N_{E}-N_{I K}\right) \times N_{E},\left(M-N_{E}-N_{I K}\right) \times N_{I K}$ and $\left(\mathrm{M}-\mathrm{N}_{\mathrm{E}}-\mathrm{N}_{\mathrm{IK}}\right) \times \mathrm{N}_{\mathrm{DK}}$ respectively, and, $\mathbf{r}_{\mathrm{E}}, \mathbf{r}_{\mathrm{IK}}$ and $\mathbf{r}_{\mathrm{DK}}$ are the equilibrium, the linear independent kinetic, and the linear dependent kinetic reaction rate subvectors of $\mathbf{r}$ with sizes $\mathrm{N}_{\mathrm{E}}, \mathrm{N}_{\mathrm{IK}}$ and $\mathrm{N}_{\mathrm{DK}}$, respectively.

As a result, the decomposition of Eq. (3) to Eq. (4) effectively reduces a set of $\mathrm{M}$ simultaneous reactive transport equations into three sets. The first set contains $\mathrm{N}_{\mathrm{E}}$ equilibrium-variable transport equations involving equilibrium reactions represented by corresponding thermodynamic equilibrium equations, the second set contains $\mathrm{N}_{\mathrm{IK}}$ kinetic-variable transport equations involving kinetic reactions only with each equation containing one and only one linearly indepen- dent kinetic reaction, and the third set contains $N_{C}=M-N_{E}$ - $\mathrm{N}_{\mathrm{IK}}$ component transport equations involving no reactions. These three sets of equations are described below.

Thermodynamic equilibrium equations for equilibrium reactions

$$
\begin{aligned}
\frac{\partial\left(\mathrm{AE}_{\mathrm{i}}\right)}{\partial \mathrm{t}}+\mathrm{L}\left(\mathrm{E}_{\mathrm{i}}^{\mathrm{m}}\right) & =\mathrm{A} \mathbf{D}_{1 \mathrm{ik}} \mathbf{r}_{\mathrm{Ek}}+\mathrm{A} \sum_{\mathrm{j}=1}^{\mathrm{N}_{\mathrm{IK}}} \boldsymbol{v}_{3 \mathrm{kj}} \mathbf{r}_{\mathrm{IKj}}+\mathrm{A} \sum_{\mathrm{j}=1}^{\mathrm{N}_{\mathrm{DK}}} \boldsymbol{v}_{4 \mathrm{kj}} \mathbf{r}_{\mathrm{DKj}}, \\
\mathbf{r}_{\mathrm{Ek}} & =\mathbf{r}_{\mathrm{Ek}}^{\mathrm{f}}-\mathbf{r}_{\mathrm{Ek}}^{\mathrm{b}}, \quad \mathrm{i} \in \mathrm{M}, \mathrm{k} \in \mathrm{N}_{\mathrm{E}}
\end{aligned}
$$

Reactive transport equations for kinetic-variables

$\frac{\partial\left(\mathrm{AE}_{\mathrm{i}}\right)}{\partial \mathrm{t}}+\mathrm{L}\left(\mathrm{E}_{\mathrm{i}}^{\mathrm{m}}\right)=\mathrm{A} \mathbf{D}_{2 \mathrm{ik}} \mathbf{r}_{\mathrm{IKk}}+\mathrm{A} \sum_{\mathrm{j}=1}^{\mathrm{N}_{\mathrm{DK}}} \boldsymbol{v}_{5 \mathrm{kj}} \mathbf{r}_{\mathrm{DK} \mathrm{j}}, \mathrm{i} \in \mathrm{M}, \mathrm{k} \in \mathrm{N}_{\mathrm{KI}}$

Transport equations for components

$\frac{\partial\left(\mathrm{AE}_{\mathrm{i}}\right)}{\partial \mathrm{t}}+\mathrm{L}\left(\mathrm{E}_{\mathrm{i}}^{\mathrm{m}}\right)=0 ;$ donating $\mathrm{T}_{\mathrm{k}}=\mathrm{E}_{\mathrm{i}}, \mathrm{i} \in \mathrm{M}, \mathrm{k} \in \mathrm{N}_{\mathrm{C}}$

where $E_{i}=\sum_{j=1, M} \mathbf{U}_{i j}^{\prime} \rho_{j} C_{j}$ is the total concentration of reaction-extent $i$ that could be an equilibrium-variable [Eq. (5)], a kinetic-variable [Eq. (6)], or a component [Eq. (7)], and $\mathrm{E}_{\mathrm{i}}^{\mathrm{m}}=\sum_{\mathrm{j}=1, \mathrm{M}} \boldsymbol{\alpha}_{\mathrm{ij}}^{\prime} \rho_{\mathrm{j}} \mathrm{C}_{\mathrm{j}}$ is the mobile part concentration of reaction-extent $\mathrm{i}$. Equations (5) through (7) constinute $(\mathrm{M}+$ $\mathrm{N})$ equations for $(\mathrm{M}+\mathrm{N})$ unknowns, which are the concentrations of $\mathrm{M}$ species and reaction rates of $\mathrm{N}$ reactions. For a kinetic equation, its rate can be explicitly formulated as a function of species concentrations. However, for an equilibrium reaction, both its forward rate, $\mathbf{r}_{\mathbf{E k}}^{\mathrm{f}}$, and backward rate, $\mathbf{r}_{\mathrm{Ek}}^{\mathrm{b}}$ are infinite, its net rate, $\mathbf{r}_{\mathrm{Ek}}=\mathbf{r}_{\mathrm{Ek}}^{\mathrm{f}}-\mathbf{r}_{\mathrm{Ek}}^{\mathrm{b}}$, cannot be explicitly formulated in terms of species concentrations. Instead, a consistent thermodynamic approach can be used to implicitly define its rate as

$\mathrm{K}_{\mathrm{k}}^{\mathrm{e}}=\sum_{\mathrm{i} \in \mathrm{M}}\left(\rho_{\mathrm{i}} \mathrm{C}_{\mathrm{ik}}\right)^{v_{\mathrm{ik}}} / \sum_{\mathrm{i} \in \mathrm{M}}\left(\rho_{\mathrm{i}} \mathrm{C}_{\mathrm{ik}}\right)^{\mu_{\mathrm{ik}}}, \quad \mathrm{k} \in \mathrm{N}_{\mathrm{E}}$ 
where $\mathrm{K}_{\mathrm{i}}^{\mathrm{e}}$ is the equilibrium constant of the k-th reaction. Once the reaction rates of all kinetic reactions are explicitly formulated, Eq. (5) is decoupled from the system of Eqs. (6), (7), and (8). It can be simply stated that Eqs. (6), (7), and (8) are solved for the concentrations of M species followed by posterior calculation of equilibrium reaction rates with Eq. (5).

To achieve efficient and robust computations, three coupling strategies to deal with reactive chemistry and five numerical options to solve the advective-dispersive transport equation were considered and introduced in details by (Zhang et al. 2008). For each time step, the advective-dispersive transport with or without reaction terms is solved first, reaction-extent by reaction-extent [e.g., Eqs. (6) and (7) yielded from complete decomposition]. Then, the reactive chemical system is solved node by node to yield concentrations of all water quality constituents (Zhang et al. 2008).

\section{DES MOINES RIVER CASE STUDY}

The water quality analysis simulation program (WASP5) is a group of conventional mechanistic water quality models capable of simulating fate and transport of water quality variables and toxic organics for aquatic systems (Ambrose et al. 1993). Various components of WASP5 have been used to study river, lake, reservoir, and estuarine issues, including ecological characterization, the effects of anthropogenic activities, and the impact of mitigation measures (Bierman and James 1995; Lung and Larson 1995; Tufford and McKellar 1999; Zheng et al. 2004).

EUTRO5 is a version of WASP5 used to simulate nutrient enrichment, eutrophication, and dissolved oxygen in aquatic environments. EUTRO5 constitutes a complex of four interacting systems: dissolved oxygen, nitrogen cycle, phosphorus cycle, and phytoplankton dynamics. It can simulate up to eight species in the water column and the benthic layer by solving 16 working equations (Table 1 ) for 16 state-variables, including: (1) ammonia $\mathrm{NH}_{3}$ and $\mathrm{NH}_{3(b)}$, (2) nitrate $\mathrm{NO}_{3}$ and $\mathrm{NO}_{3(b)}$, (3) inorganic phosphorus $\mathrm{OPO}_{4}$ and $\mathrm{OPO}_{4(\mathrm{~b})}$, (4) phytoplankton PHYT and $\mathrm{PHYT}_{(\mathrm{b})}$, (5) carbonaceous $\mathrm{CH}_{2} \mathrm{Ot}$ and $\mathrm{CH}_{2} \mathrm{Ot}_{(\mathrm{b})}$, (6) oxygen $\mathrm{O}_{2}$ and $\mathrm{O}_{2(\mathrm{~b})}$, (7) organic nitrogen $\mathrm{ONt}$ and $\mathrm{ONt}_{(\mathrm{b})}$, and (8) organic phosphorus OPt and $\mathrm{OPt}_{(\mathrm{b})}$, where ' $\mathrm{t}$ ' denotes total and subscript '(b)' denotes benthic. A total concentration is the sum of a corresponding dissolved concentration and that sorbed on sediments, for example, $\mathrm{CH}_{2} \mathrm{Ot}=\mathrm{CH}_{2} \mathrm{O}+\mathrm{CH}_{2} \mathrm{Op}, \mathrm{CH}_{2} \mathrm{Ot}_{(\mathrm{b})}$ $=\mathrm{CH}_{2} \mathrm{O}_{(\mathrm{b})}+\mathrm{CH}_{2} \mathrm{Op}_{(\mathrm{b})}, \mathrm{ONt}=\mathrm{ON}+\mathrm{ONp}, \mathrm{ONt}_{(\mathrm{b})}=\mathrm{ON}_{(\mathrm{b})}+$ $\mathrm{ONp}_{(\mathrm{b})}, \mathrm{OPt}=\mathrm{OP}+\mathrm{OPp}$, and $\mathrm{OPt}_{(\mathrm{b})}=\mathrm{OP}_{(\mathrm{b})}+\mathrm{OPp}_{(\mathrm{b})}$, where ' $p$ ' denotes particulate sorbed onto sediment. Therefore, the 16 state-variables simulated in EUTRO5 were transformed into 22 water quality constituents.

To employ the reaction-based decomposition method and the computer code we have developed (Yeh and Zhang 2005), the 16 working equations of EUTRO5 were recast in the context of a reaction network (Table 2), which includes 32 kinetic reactions and 6 equilibrium reactions involving 27 water quality constituents: $\mathrm{NH}_{3}, \mathrm{NH}_{3(\mathfrak{b})}, \mathrm{NO}_{3}, \mathrm{NO}_{3(\mathfrak{b})}, \mathrm{OPO}_{4}$, $\mathrm{OPO}_{4(\mathrm{~b})}$, PHYT, $\mathrm{PHYT}_{(\mathrm{b})}, \mathrm{CH}_{2} \mathrm{O}, \mathrm{CH}_{2} \mathrm{Op}, \mathrm{CH}_{2} \mathrm{O}_{(\mathrm{b})}, \mathrm{CH}_{2} \mathrm{Op}(\mathrm{b})$, $\mathrm{O}_{2}, \mathrm{O}_{2(\mathrm{~b})}, \mathrm{ON}, \mathrm{ONp}, \mathrm{ON}_{(\mathrm{b})}, \mathrm{ONp}_{(\mathrm{b})}, \mathrm{OP}, \mathrm{OPp}, \mathrm{OP}_{(\mathrm{b})}, \mathrm{OPp}_{(\mathrm{b})}$, $\mathrm{CO}_{2}, \mathrm{H}_{2} \mathrm{O}, \mathrm{H}^{+}, \mathrm{N}_{2}$, and $\mathrm{O}_{2(\mathrm{~g})}$. Rates of the 32 kinetic reactions used in EUTRO5 were assumed not to be affected by the last 5 constituents. Thus, the latter 5 constituents can be decoupled from the other 22. One therefore needs to simulate only 22 constituents simultaneously from a reaction based point of view. Had evidence indicated that the rate formulation of the 32 kinetic reactions also depended on the other 5 constituents, then all 27 constituents would be modeled simultaneously.

The question may be asked why EUTRO5 considers 16 rather than 22 water quality state-variables. Examination of 6 equilibrium reactions reveals that the adsorption reactions of aqueous $\mathrm{CH}_{2} \mathrm{O}, \mathrm{CH}_{2} \mathrm{O}_{(\mathrm{b})}, \mathrm{ON}, \mathrm{ON}_{(\mathrm{b})}, \mathrm{OP}$, and $\mathrm{OP}_{(\mathrm{b})}$ onto sediments were formulated as simple partition relationships. Furthermore, rate equations are only functions of the aqueous fractions of $\mathrm{CH}_{2} \mathrm{Ot}\left[=\mathrm{CH}_{2} \mathrm{O}+\mathrm{CH}_{2} \mathrm{Op}\right], \mathrm{CH}_{2} \mathrm{Ot}_{(\text {(b) }}$ [= $\left.\mathrm{CH}_{2} \mathrm{O}_{(\mathrm{b})}+\mathrm{CH}_{2} \mathrm{Op}_{(\mathrm{b})}\right], \mathrm{ONt}[=\mathrm{ON}+\mathrm{ONp}], \mathrm{ONt}_{(\mathrm{b})}\left[=\mathrm{ON}_{(\mathrm{b})}+\right.$ $\left.\mathrm{ONp}_{(b)}\right], \mathrm{OPt}[=\mathrm{OP}+\mathrm{OPp}]$, and $\mathrm{OPt}_{(\mathrm{b})}\left[=\mathrm{OP}_{(\mathrm{b})}+\mathrm{OPp}_{(\mathrm{b})}\right]$, not functions of 12 individual water quality constituents. Eliminating these 12 water quality constituents using the 6 partition equations and 6 equations defining the totals, the reaction-based approach yields 16 identical equations as those in EUTRO5. In our reaction-based approach, we prefer to model all 22 water quality constituents. This allows us, if necessary, the flexibility of more mechanistically modeling the sorption reactions and formulating the rate equations as functions of all individual constituents. During decomposition of the reaction matrix, elimination of the 6 equilibrium reactions is performed automatically rather than manually.

To demonstrate the capability of the proposed reactionbased model and to validate the application of EUTRO5 to a field problem, a case study was conducted for the Des Moines River in Iowa, USA (Fig. 2), for which the eutrophication model of WASP5 was applied successfully ( $\mathrm{Gu}$ and Dong 1998). The reaction network (Table 2) extracted from the WASP5 working equations (Table 1) was employed in the following simulation.

The study reach begins at the Des Moines Sewage Treatment Plant located upstream of water quality sampling station 6 and ends $38.6 \mathrm{~km}$ downstream at station 7. The stream flow in the river reach was highly variable over time. Severe drought conditions were experienced in central Iowa during the summer of 1977. The low flow condition in July 1977 offers an excellent opportunity to demonstrate the eutrophication process as reaction kinetics becomes more pronounced without significant source load from the drainage area of the reach. Therefore, the water quality data obtained on 13 July 1977 are used to validate the WASP5 eutrophication model using the reaction-based diagonalization 
Table 1. Original working equations of EUTRO5.

\begin{tabular}{|c|c|c|c|}
\hline No. & Variables & Notation & Working equations \\
\hline 1 & $\mathrm{NH}_{3}$ & $\mathrm{C}_{1}$ & $\frac{\partial \mathrm{C}_{1}}{\partial \mathrm{t}}=\frac{\mathrm{D}_{\mathrm{P} 1} \mathrm{a}_{\mathrm{nc}}\left(1-\mathrm{f}_{\mathrm{on}}\right) \mathrm{C}_{4}}{(\text { death })}+\frac{\mathrm{k}_{71} \Theta_{71}^{\mathrm{T}-20}\left(\frac{\mathrm{C}_{4}}{\mathrm{~K}_{\mathrm{mpc}}+\mathrm{C}_{4}}\right) \mathrm{C}_{7}}{(\text { mineralization })}-\frac{\mathrm{G}_{\mathrm{P} 1} \mathrm{a}_{\mathrm{nc}} \mathrm{P}_{\mathrm{NH}} \mathrm{C}_{4}}{(\text { growth })}-\frac{\mathrm{k}_{12} \Theta_{12}^{\mathrm{T}-20}\left(\frac{\mathrm{C}_{6}}{\mathrm{~K}_{\mathrm{NIT}}+\mathrm{C}_{6}}\right) \mathrm{C}_{1}}{\text { (nitrification })}+\frac{\frac{\mathrm{E}_{\mathrm{DIF}}}{\mathrm{A} / \mathrm{P}}\left(\mathrm{C}_{1(\mathrm{~b})}-\mathrm{C}_{1}\right)}{(\text { flux })}$ \\
\hline 2 & $\mathrm{NO}_{3}$ & $\mathrm{C}_{2}$ & $\frac{\partial \mathrm{C}_{2}}{\partial \mathrm{t}}=\frac{\mathrm{k}_{12} \Theta_{12}^{\mathrm{T}-20}\left(\frac{\mathrm{C}_{6}}{\mathrm{~K}_{\mathrm{NIT}}+\mathrm{C}_{6}}\right) \mathrm{C}_{1}}{\text { (nitrification })}-\frac{\mathrm{G}_{\mathrm{Pl}} \mathrm{a}_{\mathrm{nc}}\left(1-\mathrm{P}_{\mathrm{NH}}\right) \mathrm{C}_{4}}{(\text { growth })}-\frac{\mathrm{k}_{2 \mathrm{D}} \Theta_{2 \mathrm{D}}^{\mathrm{T}-20}\left(\frac{\mathrm{K}_{\mathrm{NO}_{3}}}{\mathrm{~K}_{\mathrm{NO}_{3}}+\mathrm{C}_{6}}\right) \mathrm{C}_{2}}{(\text { denitrification })}+\frac{\frac{\mathrm{E}_{\mathrm{DIF}}}{\mathrm{A} / \mathrm{P}}\left(\mathrm{C}_{2(\mathrm{~b})}-\mathrm{C}_{2}\right)}{(\text { flux })}$ \\
\hline 3 & $\mathrm{OPO}_{4}$ & $\mathrm{C}_{3}$ & $\frac{\partial \mathrm{C}_{3}}{\partial \mathrm{t}}=\frac{\mathrm{D}_{\mathrm{Pl}} \mathrm{a}_{\mathrm{pc}}\left(1-\mathrm{f}_{\mathrm{op}}\right) \mathrm{C}_{4}}{(\text { death })}+\frac{\mathrm{k}_{83} \Theta_{83}^{\mathrm{T}-20}\left(\frac{\mathrm{C}_{4}}{\mathrm{~K}_{\mathrm{mpc}}+\mathrm{C}_{4}}\right) \mathrm{C}_{8}}{(\text { mineralization })}-\frac{\mathrm{G}_{\mathrm{P} 1} \mathrm{a}_{\mathrm{pc}} \mathrm{C}_{4}}{(\text { growth })}+\frac{\frac{\mathrm{E}_{\mathrm{DIF}}}{\mathrm{A} / \mathrm{P}}\left(\mathrm{C}_{3(\mathrm{~b})}-\mathrm{C}_{3}\right)}{(\text { flux })}$ \\
\hline 4 & PHYT & $\mathrm{C}_{4}$ & $\frac{\partial \mathrm{C}_{4}}{\partial \mathrm{t}}=\frac{\mathrm{G}_{\mathrm{P} 1} \mathrm{C}_{4}}{(\text { growth })}-\frac{\mathrm{D}_{\mathrm{P} 1} \mathrm{C}_{4}}{(\text { death })}-\frac{\frac{\mathrm{V}_{\mathrm{s} 4}}{\mathrm{~A} / \mathrm{P}} \mathrm{C}_{4}}{(\text { settling })}$ \\
\hline 5 & $\mathrm{CH}_{2} \mathrm{Ot}$ & $\mathrm{C}_{5}$ & $\begin{aligned} \frac{\partial \mathrm{C}_{5}}{\partial \mathrm{t}}= & \frac{\mathrm{a}_{\mathrm{oc}}\left(\mathrm{K}_{\mathrm{lD}} \mathrm{C}_{4}+\mathrm{K}_{1 \mathrm{~g}} \mathrm{ZC}_{4}\right)}{(\text { death })}-\frac{\mathrm{k}_{\mathrm{D}} \Theta_{\mathrm{D}}^{\mathrm{T}-20}\left(\frac{\mathrm{C}_{6}}{\mathrm{~K}_{\mathrm{BOD}}+\mathrm{C}_{6}}\right) \mathrm{C}_{5}}{(\text { oxidation })}-\frac{\frac{\mathrm{V}_{\mathrm{s} 3}\left(1-\mathrm{f}_{\mathrm{D} 5}\right)}{\mathrm{A} / \mathrm{P}} \mathrm{C}_{5}}{(\text { settling })}-\frac{\frac{5}{4} \frac{32}{14} \mathrm{k}_{2 \mathrm{D}} \Theta_{2 \mathrm{D}}^{\mathrm{T}-20}\left(\frac{\mathrm{K}_{\mathrm{NO}_{3}}}{\mathrm{~K}_{\mathrm{NO}_{3}}+\mathrm{C}_{6}}\right) \mathrm{C}_{2}}{(\text { denitrification })} \\
& \frac{\mathrm{V}_{\mathrm{R} 3}\left(1-\mathrm{f}_{\mathrm{D} 5(\mathrm{~b})}\right)}{\mathrm{A} / \mathrm{P}} \mathrm{C}_{5(\mathrm{~b})} \frac{\mathrm{E}_{\mathrm{DF}}}{\mathrm{h}_{\mathrm{b}} \mathrm{A} / \mathrm{P}}\left(\mathrm{C}_{5} \mathrm{f}_{\mathrm{D} 5}-\mathrm{C}_{5(\mathrm{~b})} \mathrm{f}_{\mathrm{D} 5(\mathrm{~b})}\right)\end{aligned}$ \\
\hline 6 & $\mathrm{O}_{2}$ & $\mathrm{C}_{6}$ & $\begin{aligned} \frac{\partial \mathrm{C}_{6}}{\partial \mathrm{t}}= & \frac{\mathrm{k}_{2} \Theta_{2}^{\mathrm{T}-20}\left(\mathrm{C}_{\mathrm{s}}-\mathrm{C}_{6}\right)}{(\text { reaeration })}-\frac{\mathrm{k}_{\mathrm{d}} \Theta_{\mathrm{d}}^{\mathrm{T}-20}\left(\frac{\mathrm{C}_{6}}{\mathrm{~K}_{\mathrm{BOD}}+\mathrm{C}_{6}}\right) \mathrm{C}_{5}}{(\text { oxidation })}-\frac{\frac{64}{14} \mathrm{k}_{12} \Theta_{12}^{\mathrm{T}-20}\left(\frac{\mathrm{C}_{6}}{\mathrm{~K}_{\mathrm{NIT}}+\mathrm{C}_{6}}\right) \mathrm{C}_{1}}{\text { (nitrification) }}+\frac{\mathrm{G}_{\mathrm{P} 1}\left[\frac{32}{12}+\frac{48}{14} \frac{14}{12}\left(1-\mathrm{P}_{\mathrm{NH} 3}\right)\right] \mathrm{C}_{4}}{\text { (phytoplankton growth) }} \\
& -\frac{\frac{32}{12} \mathrm{k}_{1 \mathrm{R}} \Theta_{1 \mathrm{R}}^{\mathrm{T}-20} \mathrm{C}_{4}}{(\text { respiration })}-\frac{\frac{\mathrm{E}_{\mathrm{DIF}}}{\mathrm{h}_{\mathrm{b}} \mathrm{A} / \mathrm{P}}\left(\mathrm{C}_{6}-\mathrm{C}_{6(\mathrm{~b})}\right)}{(\text { diffusion })}\end{aligned}$ \\
\hline 7 & $\mathrm{ONt}$ & $\mathrm{C}_{7}$ & $\frac{\partial \mathrm{C}_{7}}{\partial \mathrm{t}}=\frac{\mathrm{D}_{\mathrm{P} 1} \mathrm{a}_{\mathrm{nc}} \mathrm{f}_{\mathrm{on}} \mathrm{C}_{4}}{(\text { death })}-\frac{\mathrm{k}_{71} \Theta_{71}^{\mathrm{T}-20}\left(\frac{\mathrm{C}_{4}}{\mathrm{~K}_{\mathrm{mPc}}+\mathrm{C}_{4}}\right) \mathrm{C}_{7}}{(\text { mineralization })}-\frac{\frac{\mathrm{V}_{\mathrm{s} 3}\left(1-\mathrm{f}_{\mathrm{D} 7}\right)}{\mathrm{A} / \mathrm{P}} \mathrm{C}_{7}}{(\text { settling })}+\frac{\frac{\mathrm{E}_{\mathrm{DIF}}}{\mathrm{A} / \mathrm{P}}\left(\mathrm{C}_{7 \mathrm{~b}(\mathrm{~b})} \mathrm{f}_{\mathrm{D} 7 \mathrm{~b})}-\mathrm{C}_{7} \mathrm{f}_{\mathrm{D} 7}\right)}{(\text { flux })}$ \\
\hline 8 & $\mathrm{OPt}$ & $\mathrm{C}_{8}$ & $\frac{\partial \mathrm{C}_{8}}{\partial \mathrm{t}}=\frac{\mathrm{D}_{\mathrm{P} 1} \mathrm{a}_{\mathrm{pc}} \mathrm{f}_{\mathrm{op}} \mathrm{C}_{4}}{(\text { death })}-\frac{\mathrm{k}_{83} \Theta_{83}^{\mathrm{T}-20}\left(\frac{\mathrm{C}_{4}}{\mathrm{~K}_{\mathrm{mPc}}+\mathrm{C}_{4}}\right) \mathrm{C}_{8}}{(\text { mineralization })}-\frac{\frac{\mathrm{V}_{\mathrm{s} 3}\left(1-\mathrm{f}_{\mathrm{D} 8}\right)}{\mathrm{A} / \mathrm{P}} \mathrm{C}_{8}}{(\text { settling })}+\frac{\frac{\mathrm{E}_{\mathrm{DIF}}}{\mathrm{A} / \mathrm{P}}\left(\mathrm{C}_{8(\mathrm{~b})} \mathrm{f}_{\mathrm{D} 8(\mathrm{~b})}-\mathrm{C}_{8} \mathrm{f}_{\mathrm{D} 8}\right)}{(\text { flux })}$ \\
\hline 9 & $\mathrm{NH}_{3(\mathrm{~b})}$ & $\mathrm{C}_{1(\mathrm{~b})}$ & $\frac{\partial C_{1(b)}}{\partial t}=\frac{k_{\mathrm{PZD}} \Theta_{\mathrm{PZD}}^{\mathrm{T}-20} \mathrm{a}_{\mathrm{nc}}\left(1-\mathrm{f}_{\mathrm{on}(\mathrm{b})}\right) \mathrm{C}_{4(\mathrm{~b})}}{(\text { algal decomposition })}+\frac{\mathrm{k}_{\mathrm{OND}} \Theta_{\mathrm{OND}}^{\mathrm{T}-20} \mathrm{f}_{\mathrm{D} 7(\mathrm{~b})} \mathrm{C}_{7(\mathrm{~b})}}{(\text { mineralization })}-\frac{\frac{\mathrm{E}_{\mathrm{DIF}}}{\mathrm{h}_{\mathrm{b}}}\left(\mathrm{C}_{1(\mathrm{~b})}-\mathrm{C}_{1}\right)}{(\text { flux })}$ \\
\hline 10 & $\mathrm{NO}_{3(\mathrm{~b})}$ & $\mathrm{C}_{2(\mathrm{~b})}$ & $\frac{\partial C_{2(b)}}{\partial t}=-\frac{k_{2 D} \Theta_{2 D}^{T-20} C_{2(b)}}{(\text { denitrification })}-\frac{\frac{E_{D I F}}{h_{b}}\left(C_{2(b)}-C_{2}\right)}{(\text { flux })}$ \\
\hline 11 & $\mathrm{OPO}_{4(\mathrm{~b})}$ & $\mathrm{C}_{3(\mathrm{~b})}$ & $\frac{\partial C_{3(b)}}{\partial t}=\frac{k_{P Z D} \Theta_{\mathrm{PZD}}^{\mathrm{T}-20} a_{\mathrm{pc}}\left(1-\mathrm{f}_{\mathrm{op}(\mathrm{b})}\right) \mathrm{C}_{4(\mathrm{~b})}}{(\text { algal decomposition })}+\frac{\mathrm{k}_{\mathrm{OPD}} \Theta_{\mathrm{OPD}}^{\mathrm{T}-20} \mathrm{f}_{\mathrm{D} 8(\mathrm{~b})} \mathrm{C}_{8(\mathrm{~b})}}{(\text { mineralization })}-\frac{\frac{\mathrm{E}_{\mathrm{DIF}}}{\mathrm{h}_{\mathrm{b}}}\left(\mathrm{C}_{3(\mathrm{~b})}-\mathrm{C}_{3}\right)}{(\text { flux })}$ \\
\hline 12 & $\mathrm{PHYT}_{(\mathrm{b})}$ & $\mathrm{C}_{4(\mathrm{~b})}$ & $\frac{\partial \mathrm{C}_{4(\mathrm{~b})}}{\partial \mathrm{t}}=\frac{\mathrm{k}_{\mathrm{PZD}} \Theta_{\mathrm{PZD}}^{\mathrm{T}-20} \mathrm{C}_{4(\mathrm{~b})}}{(\text { algal decomposition })}+\frac{\frac{\mathrm{V}_{\mathrm{s4}}}{\mathrm{h}_{\mathrm{b}}} \mathrm{C}_{4}}{(\text { settling })}$ \\
\hline 13 & $\mathrm{CH}_{2} \mathrm{Ot}_{(\mathrm{b})}$ & $\mathrm{C}_{5(\mathrm{~b})}$ & 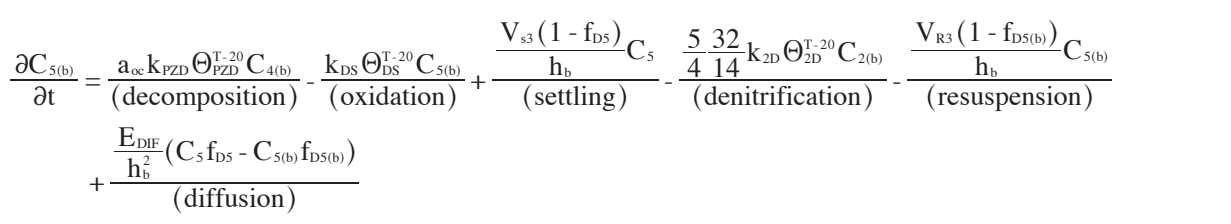 \\
\hline 14 & $\mathrm{O}_{2(\mathrm{~b})}$ & $\mathrm{C}_{6(\mathrm{~b})}$ & $\frac{\partial C_{6(b)}}{\partial t}=-\frac{k_{D S} \Theta_{D S}^{T-20} C_{5(b)}}{(\text { oxidation })}+\frac{\frac{E_{D D F}}{h_{b}^{2}}\left(C_{6}-C_{6(b)}\right)}{(\text { diffusion })}$ \\
\hline 15 & $\mathrm{ONt}_{(\mathrm{b})}$ & $\mathrm{C}_{7(\mathrm{~b})}$ & $\frac{\partial C_{7(b)}}{\partial t}=\frac{k_{\mathrm{PZD}} \Theta_{\mathrm{PZD}}^{\mathrm{T}-20} \mathrm{a}_{\mathrm{nc}} \mathrm{f}_{\mathrm{on}(\mathrm{b})} \mathrm{C}_{4(\mathrm{~b})}}{(\text { algal decomposition })}-\frac{\mathrm{k}_{\mathrm{OND}} \Theta_{\mathrm{OND}}^{\mathrm{T}-20} \mathrm{f}_{\mathrm{D} 7(\mathrm{~b})} \mathrm{C}_{7(\mathrm{~b})}}{(\text { mineralization })}+\frac{\frac{\mathrm{V}_{\mathrm{s3}}\left(1-\mathrm{f}_{\mathrm{D} 7}\right)}{\mathrm{h}_{\mathrm{b}}} \mathrm{C}_{7}}{(\text { settling })}-\frac{\frac{\mathrm{E}_{\mathrm{DIF}}}{\mathrm{h}_{\mathrm{b}}}\left(\mathrm{C}_{7(\mathrm{~b})} \mathrm{f}_{\mathrm{D} 7 \mathrm{~b})}-\mathrm{C}_{7} \mathrm{f}_{\mathrm{D} 7}\right)}{(\text { flux })}$ \\
\hline 16 & $\mathrm{OPt}_{(\mathrm{b})}$ & $\mathrm{C}_{8(\mathrm{~b})}$ & $\frac{\partial C_{8(b)}}{\partial t}=\frac{k_{P Z D} \Theta_{P Z D}^{T-20} a_{p c} f_{o p(b)} C_{4(b)}}{(\text { algal decomposition })}-\frac{k_{O P D} \Theta_{O P D}^{T-20} f_{D 8(b)} C_{8(b)}}{\text { (mineralization) }}+\frac{\frac{V_{s 3}\left(1-f_{D 8}\right)}{h_{b}} C_{8}}{(\text { settling })}-\frac{\frac{E_{D D F}}{h_{b}}\left(C_{8(b)} f_{D 8(b)}-C_{8} f_{D 8}\right)}{(\text { flux })}$ \\
\hline
\end{tabular}


Table 2. Reaction network recast from EUTRO5 working equations.

\begin{tabular}{|c|c|c|c|}
\hline No. & Mechanism & Reaction & Reaction Rate \\
\hline K1 & PHYT growth & $\mathrm{a}_{\mathrm{nc}} \mathrm{NH}_{3}+\mathrm{a}_{\mathrm{pc}} \mathrm{OPO}_{4}+\mathrm{CO}_{2}+\mathrm{H}_{2} \mathrm{O} \rightarrow \mathrm{PHYT}+\frac{32}{12} \mathrm{O}_{2}$ & $\mathrm{R}_{1}=\mathrm{G}_{\mathrm{pl}} \mathrm{C}_{7}$ \\
\hline $\mathrm{K} 2$ & PHYT growth related nitrate reduction & $\mathrm{a}_{\mathrm{nc}} \mathrm{NO}_{3} \rightarrow \mathrm{a}_{\mathrm{nc}} \mathrm{NH}_{3}+\frac{48}{12} \mathrm{O}_{2}$ & $\mathrm{R}_{2}=\left(1-\mathrm{P}_{\mathrm{NH}}\right) \mathrm{G}_{\mathrm{p} 1} \mathrm{C}_{7}$ \\
\hline K3 & PHYT death-endogenous respiration & $\mathrm{PHYT}+\frac{32}{12} \mathrm{O}_{2} \rightarrow \mathrm{CO}_{2}+\mathrm{H}_{2} \mathrm{O}+\mathrm{a}_{\mathrm{nc}} \mathrm{ON}+\mathrm{a}_{\mathrm{pc}} \mathrm{OP}$ & $\mathrm{R}_{3}=\mathrm{k}_{\mathrm{lr}} \Theta_{\mathrm{lr}}^{\mathrm{T}-20} \mathrm{C}_{7}$ \\
\hline K4 & PHYT death-parasitization & $\mathrm{PHYT} \rightarrow \mathrm{a}_{\mathrm{oc}} \mathrm{CH}_{2} \mathrm{O}+\mathrm{a}_{\mathrm{nc}} \mathrm{ON}+\mathrm{a}_{\mathrm{pc}} \mathrm{OP}$ & $\mathrm{R}_{4}=\mathrm{k}_{1 \mathrm{~d}} \mathrm{C}_{7}$ \\
\hline K5 & PHYT death-herbivorous grazing & $\mathrm{PHYT} \rightarrow \mathrm{a}_{\mathrm{oc}} \mathrm{CH}_{2} \mathrm{O}+\mathrm{a}_{\mathrm{nc}} \mathrm{ON}+\mathrm{a}_{\mathrm{pc}} \mathrm{OP}$ & $\mathrm{R}_{5}=\mathrm{k}_{1 \mathrm{~g}} \mathrm{ZC}_{7}$ \\
\hline K6 & PHYT death-promoted oxidation of ON & $\mathrm{a}_{\mathrm{nc}} \mathrm{ON} \rightarrow \mathrm{a}_{\mathrm{nc}} \mathrm{NH}_{3}$ & $\mathrm{R}_{6}=\left(1-\mathrm{f}_{\mathrm{on}}\right)\left(\mathrm{k}_{\mathrm{lr}} \Theta_{\mathrm{lr}}^{\mathrm{T}-20} \mathrm{C}_{7}+\mathrm{k}_{1 \mathrm{~d}} \mathrm{C}_{7}+\mathrm{k}_{\mathrm{lg}} \mathrm{ZC}_{7}\right)$ \\
\hline K7 & PHYT death-promoted oxidation of OP & $\mathrm{a}_{\mathrm{pc}} \mathrm{OP} \rightarrow \mathrm{a}_{\mathrm{pc}} \mathrm{OPO}_{4}$ & $\mathrm{R}_{7}=\left(1-\mathrm{f}_{\mathrm{op}}\right)\left(\mathrm{k}_{\mathrm{lr}} \Theta_{\mathrm{lr}}^{\mathrm{T}-20} \mathrm{C}_{7}+\mathrm{k}_{\mathrm{ld}} \mathrm{C}_{7}+\mathrm{k}_{\mathrm{lg}} \mathrm{ZC}_{7}\right)$ \\
\hline K8 & Benthic PHYT decomposition & $\mathrm{PHYT}_{(b)} \rightarrow \mathrm{a}_{\mathrm{oc}} \mathrm{CH}_{2} \mathrm{O}_{(\mathrm{b})}+\mathrm{a}_{\mathrm{nc}} \mathrm{ON}_{(\mathrm{b})}+\mathrm{a}_{\mathrm{pc}} \mathrm{OP}_{(\mathrm{b})}$ & $\mathrm{R}_{8}=\mathrm{k}_{\mathrm{PZD}} \Theta_{\mathrm{PZD}}^{\mathrm{T}-20} \mathrm{C}_{8}$ \\
\hline K9 & $\begin{array}{l}\mathrm{PHYT}_{(\mathrm{b})} \text { decomposition promoted } \\
\text { oxidation of } \mathrm{ON}_{(\mathrm{b})}\end{array}$ & $\mathrm{a}_{\mathrm{nc}} \mathrm{ON}_{(\mathrm{b})} \rightarrow \mathrm{a}_{\mathrm{nc}} \mathrm{NH}_{3(\mathrm{~b})}$ & $\mathrm{R}_{9}=\left(1-\mathrm{f}_{\text {on(bed) }}\right) \mathrm{k}_{\mathrm{PZD}} \Theta_{\mathrm{PZD}}^{\mathrm{T}-20} \mathrm{C}_{8} \cdot \mathrm{h}_{\mathrm{b}} \mathrm{P} / \mathrm{A}$ \\
\hline K10 & $\begin{array}{l}\mathrm{PHYT}_{(\mathrm{b})} \text { decomposition Promoted } \\
\text { oxidation of } \mathrm{OP}_{(\mathrm{b})}\end{array}$ & $\mathrm{a}_{\mathrm{pc}} \mathrm{OP}_{(\mathrm{b})} \rightarrow \mathrm{a}_{\mathrm{pc}} \mathrm{OPO}_{4(\mathrm{~b})}$ & $\mathrm{R}_{10}=\left(1-\mathrm{f}_{\text {op }(\text { bed })}\right) \mathrm{k}_{\mathrm{PZD}} \Theta_{\mathrm{PZD}}^{\mathrm{T}-20} \mathrm{C}_{8} \cdot \mathrm{h}_{\mathrm{b}} \mathrm{P} / \mathrm{A}$ \\
\hline K11 & Phytoplankton settling & $\mathrm{PHYT} \rightarrow \mathrm{PHYT}_{(b)}$ & $\mathrm{R}_{11}=\frac{\mathrm{V}_{\mathrm{s}}}{\mathrm{h}_{\mathrm{b}}} \mathrm{C}_{7} \cdot \mathrm{h}_{\mathrm{b}} \mathrm{P} / \mathrm{A}$ \\
\hline K12 & Re-aeration & $\mathrm{O}_{2(\mathrm{~g})} \rightarrow \mathrm{O}_{2}$ & $\mathrm{R}_{12}=\mathrm{k}_{2} \Theta_{\mathrm{a}}^{\mathrm{T}-20}\left(\mathrm{C}_{\mathrm{s}}-\mathrm{C}_{13}\right)$ \\
\hline K13 & Oxygen diffusion & $\mathrm{O}_{2} \rightarrow \mathrm{O}_{2(\mathrm{~b})}$ & $\mathrm{R}_{13}=\frac{\mathrm{E}_{\mathrm{DIF}}}{\mathrm{h}_{\mathrm{b}}^{2}}\left(\mathrm{C}_{13}-\mathrm{C}_{14}\right) \cdot \mathrm{h}_{\mathrm{b}} \mathrm{P} / \mathrm{A}$ \\
\hline K14 & Carbonaceous oxidation & $\mathrm{CH}_{2} \mathrm{O}+\mathrm{O}_{2} \rightarrow \mathrm{CO}_{2}+\mathrm{H}_{2} \mathrm{O}$ & $\mathrm{R}_{14}=\mathrm{k}_{\mathrm{d}} \Theta_{\mathrm{d}}^{\mathrm{T}-20}\left(\frac{\mathrm{C}_{13}}{\mathrm{~K}_{\mathrm{BOD}}+\mathrm{C}_{13}}\right)\left(\mathrm{C}_{9}+\mathrm{C}_{10}\right)$ \\
\hline K15 & Benthic carbonaceous oxidation & $\mathrm{CH}_{2} \mathrm{O}_{(\mathrm{b})}+\mathrm{O}_{2(\mathrm{~b})} \rightarrow \mathrm{CO}_{2}+\mathrm{H}_{2} \mathrm{O}$ & $\mathrm{R}_{15}=\mathrm{k}_{\mathrm{DS}} \Theta_{\mathrm{DS}}^{\mathrm{T}-20}\left(\mathrm{C}_{11}+\mathrm{C}_{12}\right) \cdot \mathrm{h}_{\mathrm{b}} \mathrm{P} / \mathrm{A}$ \\
\hline K16 & Carbonaceous settling & $\mathrm{CH}_{2} \mathrm{O}_{(\mathrm{p})} \rightarrow \mathrm{CH}_{2} \mathrm{O}_{(\mathrm{bp})}$ & $\mathrm{R}_{16}=\frac{\mathrm{V}_{\mathrm{S} 3}}{\mathrm{~h}_{\mathrm{b}}} \mathrm{C}_{10} \cdot \mathrm{h}_{\mathrm{b}} \mathrm{P} / \mathrm{A}$ \\
\hline K17 & Carbonaceous re-suspension & $\mathrm{CH}_{2} \mathrm{O}_{(\mathrm{pp})} \rightarrow \mathrm{CH}_{2} \mathrm{O}_{(\mathrm{p})}$ & $\mathrm{R}_{17}=\frac{\mathrm{V}_{\mathrm{R} 3}}{\mathrm{~h}_{\mathrm{b}}} \mathrm{C}_{12} \cdot \mathrm{h}_{\mathrm{b}} \mathrm{P} / \mathrm{A}$ \\
\hline K18 & Carbonaceous diffusion & $\mathrm{CH}_{2} \mathrm{O} \rightarrow \mathrm{CH}_{2} \mathrm{O}_{(b)}$ & $\mathrm{R}_{18}=\frac{\mathrm{E}_{\mathrm{DIF}}}{\mathrm{h}_{\mathrm{b}}^{2}}\left(\mathrm{C}_{9}-\mathrm{C}_{11}\right) \cdot \mathrm{h}_{\mathrm{b}} \mathrm{P} / \mathrm{A}$ \\
\hline K19 & Nitrogen mineralization & $\mathrm{ON} \rightarrow \mathrm{NH}_{3}$ & $\mathrm{R}_{19}=\mathrm{k}_{71} \Theta_{71}^{\mathrm{T}-20}\left(\frac{\mathrm{C}_{7}}{\mathrm{~K}_{\mathrm{mPc}}+\mathrm{C}_{7}}\right)\left(\mathrm{C}_{15}+\mathrm{C}_{16}\right)$ \\
\hline K20 & Nitrification & $\mathrm{NH}_{3}+\frac{64}{14} \mathrm{O}_{2} \rightarrow \mathrm{NO}_{3}^{-}+\mathrm{H}_{2} \mathrm{O}+\mathrm{H}^{+}$ & $\mathrm{R}_{20}=\mathrm{k}_{12} \Theta_{12}^{\mathrm{T}-20}\left(\frac{\mathrm{C}_{13}}{\mathrm{~K}_{\mathrm{NIT}}+\mathrm{C}_{13}}\right) \mathrm{C}_{1}$ \\
\hline K21 & De-nitrification & $\frac{5}{4} \mathrm{CH}_{2} \mathrm{O}+\frac{14}{32} \mathrm{NO}_{3}+\mathrm{H}^{+} \rightarrow \frac{5}{4} \mathrm{CO}_{2}+\frac{1}{2} \mathrm{~N}_{2}+\frac{7}{4} \mathrm{H}_{2} \mathrm{O}$ & $\mathrm{R}_{21}=\mathrm{k}_{2 \mathrm{D}} \Theta_{2 \mathrm{D}}^{\mathrm{T}-2 \mathrm{O}}\left(\frac{\mathrm{K}_{\mathrm{NO}_{3}}}{\mathrm{~K}_{\mathrm{NO}_{3}}+\mathrm{C}_{13}}\right) \mathrm{C}_{3} \cdot \frac{32}{14}$ \\
\hline $\mathrm{K} 22$ & Benthic nitrogen mineralization & $\mathrm{ON}_{(b)} \rightarrow \mathrm{NH}_{3(\mathrm{~b})}$ & $\mathrm{R}_{22}=\mathrm{k}_{\mathrm{OND}} \Theta_{\mathrm{OND}}^{\mathrm{T}-20} \mathrm{C}_{17} \cdot \mathrm{h}_{\mathrm{b}} \mathrm{P} / \mathrm{A}$ \\
\hline K23 & Benthic de-nitrification & $\frac{5}{4} \mathrm{CH}_{2} \mathrm{O}_{(b)}+\frac{14}{32} \mathrm{NO}_{3(\mathrm{~b})}+\mathrm{H}^{+} \rightarrow \frac{5}{4} \mathrm{CO}_{2}+\frac{1}{2} \mathrm{~N}_{2}+\frac{7}{4} \mathrm{H}_{2} \mathrm{O}$ & $\mathrm{R}_{23}=\mathrm{k}_{2 \mathrm{D}} \Theta_{2 \mathrm{D}}^{\mathrm{T}-20} \mathrm{C}_{4} \cdot \frac{32}{14} \cdot \mathrm{h}_{\mathrm{b}} \mathrm{P} / \mathrm{A}$ \\
\hline K24 & Ammonia flux & $\mathrm{NH}_{3(b)} \rightarrow \mathrm{NH}_{3}$ & $\mathrm{R}_{24}=\frac{\mathrm{E}_{\mathrm{DIF}}}{\mathrm{h}_{\mathrm{b}}}\left(\mathrm{C}_{2}-\mathrm{C}_{1}\right) \cdot \mathrm{h}_{\mathrm{b}} \mathrm{P} / \mathrm{A}$ \\
\hline K25 & Nitrate flux & $\mathrm{NO}_{3(b)} \rightarrow \mathrm{NO}_{3}$ & $\mathrm{R}_{25}=\frac{\mathrm{E}_{\mathrm{DF}}}{\mathrm{h}_{\mathrm{b}}}\left(\mathrm{C}_{4}-\mathrm{C}_{3}\right) \cdot \mathrm{h}_{\mathrm{b}} \mathrm{P} / \mathrm{A}$ \\
\hline K26 & Organic nitrogen settling & $\mathrm{ON}_{(\mathrm{p})} \rightarrow \mathrm{ON}_{(\mathrm{bp})}$ & $\mathrm{R}_{26}=\frac{\mathrm{V}_{\mathrm{S3}}}{\mathrm{h}_{\mathrm{b}}} \mathrm{C}_{16} \cdot \mathrm{h}_{\mathrm{b}} \mathrm{P} / \mathrm{A}$ \\
\hline K27 & Organic nitrogen flux & $\mathrm{ON}_{(b)} \rightarrow \mathrm{ON}$ & $\mathrm{R}_{27}=\frac{\mathrm{E}_{\mathrm{DIF}}}{\mathrm{h}_{\mathrm{b}}}\left(\mathrm{C}_{17}-\mathrm{C}_{15}\right) \cdot \mathrm{h}_{\mathrm{b}} \mathrm{P} / \mathrm{A}$ \\
\hline K28 & Phosphorous mineralization & $\mathrm{OP} \rightarrow \mathrm{OPO}_{4}$ & $\mathrm{R}_{28}=\mathrm{k}_{83} \Theta_{83}^{\mathrm{T}-20}\left(\frac{\mathrm{C}_{7}}{\mathrm{~K}_{\mathrm{mpc}}+\mathrm{C}_{7}}\right)\left(\mathrm{C}_{19}+\mathrm{C}_{20}\right)$ \\
\hline K29 & Benthic phosphorous mineralization & $\mathrm{OP}_{(\mathrm{b})} \rightarrow \mathrm{OPO}_{4(\mathrm{~b})}$ & $\mathrm{R}_{29}=\mathrm{k}_{\mathrm{OPD}} \Theta_{\mathrm{OPD}}^{\mathrm{T}-20} \mathrm{C}_{21} \cdot \mathrm{h}_{\mathrm{b}} \mathrm{P} / \mathrm{A}$ \\
\hline
\end{tabular}


Table 2. (Continued)

\begin{tabular}{|c|c|c|c|}
\hline No. & Mechanism & Reaction & Reaction Rate \\
\hline K30 & Phosphorous flux & $\mathrm{OPO}_{4(\mathrm{~b})} \rightarrow \mathrm{OPO}_{4}$ & $\mathrm{R}_{30}=\frac{\mathrm{E}_{\mathrm{DIF}}}{\mathrm{h}_{\mathrm{b}}}\left(\mathrm{C}_{6}-\mathrm{C}_{5}\right) \cdot \mathrm{h}_{\mathrm{b}} \mathrm{P} / \mathrm{A}$ \\
\hline K31 & Organic phosphorous setting & $\mathrm{OP}_{(\mathrm{p})} \rightarrow \mathrm{OP}_{(\mathrm{pp})}$ & $\mathrm{R}_{31}=\frac{\mathrm{V}_{\mathrm{S3}}}{\mathrm{h}_{\mathrm{b}}} \mathrm{C}_{20} \cdot \mathrm{h}_{\mathrm{b}} \mathrm{P} / \mathrm{A}$ \\
\hline K32 & Organic phosphorous flux & $\mathrm{OP}_{(b)} \rightarrow \mathrm{OP}$ & $\mathrm{R}_{32}=\frac{\mathrm{E}_{\mathrm{DIF}}}{\mathrm{h}_{\mathrm{b}}}\left(\mathrm{C}_{21}-\mathrm{C}_{19}\right) \cdot \mathrm{h}_{\mathrm{b}} \mathrm{P} / \mathrm{A}$ \\
\hline E1 & Carbonaceous sorption & $\mathrm{CH}_{2} \mathrm{O} \rightarrow \mathrm{CH}_{2} \mathrm{O}_{(\mathrm{p})}$ & $\mathrm{f}_{\mathrm{D} 5}=\frac{\mathrm{C}_{9}}{\mathrm{C}_{9}+\mathrm{C}_{10}}$ \\
\hline E2 & Organic nitrogen sorption & $\mathrm{ON} \rightarrow \mathrm{ON}_{(\mathrm{p})}$ & $f_{D 7}=\frac{C_{15}}{C_{15}+C_{16}}$ \\
\hline E3 & Organic phosphorous sorption & $\mathrm{OP} \rightarrow \mathrm{OP}_{(\mathrm{p})}$ & $f_{D 8}=\frac{C_{19}}{C_{19}+C_{20}}$ \\
\hline E4 & Benthic carbonaceous sorption & $\mathrm{CH}_{2} \mathrm{O}_{(\mathrm{b})} \rightarrow \mathrm{CH}_{2} \mathrm{O}_{(\mathrm{bp})}$ & $f_{D S(\text { bed })}=\frac{C_{11}}{C_{11}+C_{12}}$ \\
\hline E5 & Benthic organic nitrogen sorption & $\mathrm{ON}_{(\mathrm{b})} \rightarrow \mathrm{ON}_{(\mathrm{bp})}$ & $f_{D 7 \text { (bed) }}=\frac{C_{17}}{C_{17}+C_{18}}$ \\
\hline E6 & Benthic organic phosphorous sorption & $\mathrm{OP}_{(b)} \rightarrow \mathrm{OP}_{(\mathrm{bp})}$ & $f_{\mathrm{D} 8 \text { (bed) }}=\frac{\mathrm{C}_{21}}{\mathrm{C}_{21}+\mathrm{C}_{22}}$ \\
\hline
\end{tabular}

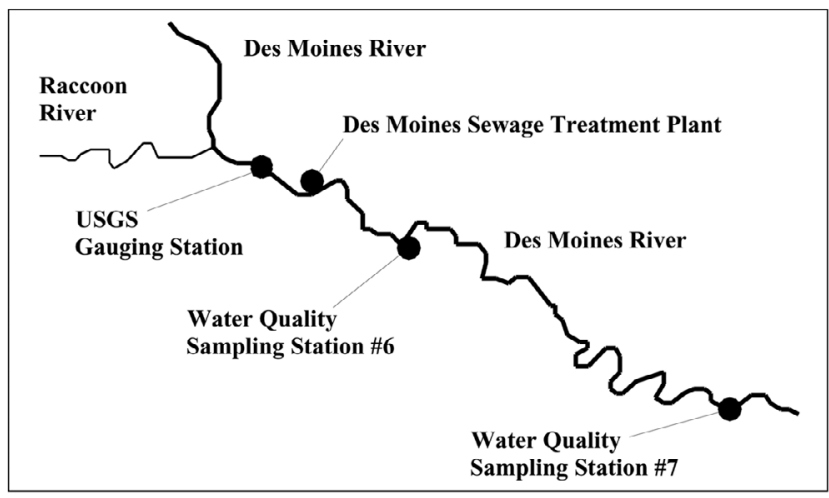

Fig. 2. The Des Moines River study area, Iowa, USA.

approach presented here. Prior to this, low stream flow conditions of about $2.5 \mathrm{~m}^{3} \mathrm{~s}^{-1}$ lasted for 6 days (Fig. 3).

The $38.6 \mathrm{~km}$ reach was assumed to have a triangular shape cross-section with side slope of 1:22.9. It was discretized into 24 elements each with a length of $1609 \mathrm{~m}$. The calculated water depth was $2.59 \mathrm{~m}$ and the flow velocity was $0.0326 \mathrm{~m} \mathrm{~s}^{-1}$. According to monitoring station observations, the water temperature was $27.5^{\circ} \mathrm{C}$, suspended sediment concentration SS was $35 \mathrm{~g} \mathrm{~m}^{-3}$, and bed sediment concentration BS was $3.26 \mathrm{~g} \mathrm{~m}^{-2}$, which are regarded as uniform throughout the reach. It was assumed that the density of column water $\rho_{\mathrm{w}}=1.0 \mathrm{~kg} \mathrm{~L}^{-1}$, density of bed water $\rho_{\mathrm{wb}}=1.0 \mathrm{~kg} \mathrm{~L}^{-1}$, bed depth $h_{b}=0.1 \mathrm{~m}$, and porosity of the bed sediment $\theta_{b}=$ 0.5. A Dirichlet boundary condition was applied at the upstream boundary. A out flow variable boundary condition was applied at the downstream boundary. Table 3 shows initial concentrations of all water quality constituents and Dirichlet boundary concentrations of mobile constituents assigned according to the water quality data obtained at a
US Geological Survey gauging station located $3.5 \mathrm{~km}$ upstream (Fig. 2). Longitudinal dispersivity was assumed to be $100 \mathrm{~m}$. A 6-day simulation was performed with a fixed time-step size of 0.5 day. Figure 4 plots the in stream concentration of DO, BOD and total ammonia as nitrogen at $\mathrm{t}=$ 6 days when field data are available. The simulated DO, BOD, and ammonia nitrogen concentration profiles agree well with field measurements. The various reaction rate parameters were determined by the typical value of the parameter or the median of the normal range given by (Ambrose et al. 1993), except that the value of carbonaceous oxidation rate coefficient kd was assigned to 0.16 day $^{-1}$ at the lower limit of the value range $0.16-0.21$ day $^{-1}$ because the model slightly under estimated both BOD and DO using the median value of 0.185 day $^{-1}$.

\section{PHYSICAL INTERPRETATION OF REACTION MATRIX DECOMPOSITION}

The general diagonalization paradigm through matrix decomposition provides a protocol for formulating rate expressions and investigating assumptions and limitations of the employed reaction model. To illustrate this, consider a simplified EUTRO5 reaction network in a well-mixed system that includes eutrophication without taking into account sediment-biogeochemical interactions and advection-dispersion. This simplification will facilitate discussion without loss of generality.

The reaction network for the simplified system is given in Table 4, which includes 13 kinetic reactions involving 13 water quality constituents: $\mathrm{NH}_{3}, \mathrm{NO}_{3}, \mathrm{OPO}_{4}$, $\mathrm{PHYT}, \mathrm{CH}_{2} \mathrm{O}$, $\mathrm{O}_{2}$, ON, OP, $\mathrm{CO}_{2}, \mathrm{H}_{2} \mathrm{O}, \mathrm{H}^{+}, \mathrm{N}_{2}$, and $\mathrm{O}_{2(\mathrm{~g})}$. Substitution of this reaction network into Eq. (1), the governing equation for a well-mixed system, results in 13 ordinary differential 
equations for 13 water quality constituents. The complete decomposition of the reaction matrix for the 13 constituents results in a set of 8 kinetic-variable equations [Eqs. (1) through (7) and Eq. (13) in Table 5] and 5 component equations [Eqs. (8) through (12) in Table 5]. If we substitute Eq. (13) into Eqs. (1) and (6) in Table 5, the resulting first 8 equations are then decoupled from the last 5 equations. The decomposition approach offers the advantage that the conservation of phosphorus is explicitly enforced when these eight equations are solved for the 8 water quality constituents

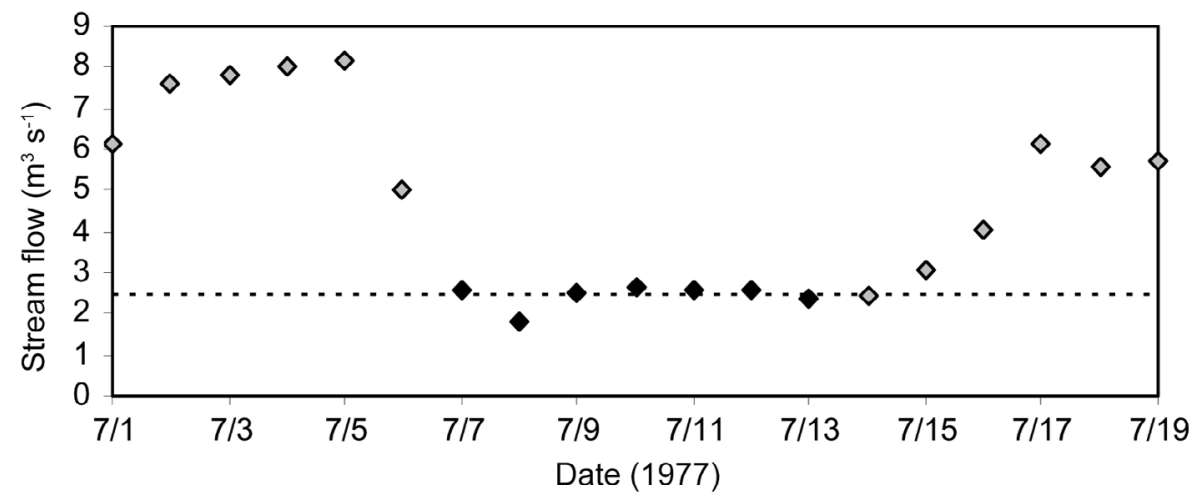

Fig. 3. The reach streamflow under study at the USGS Gauging Station during the 1977 summer drought.

Table 3. Chemical constituents simulated in the Des Moines River case study.

\begin{tabular}{|c|c|c|c|c|}
\hline Chemical constituent & Note & $\rho_{\mathrm{i}}$ & Initial concentration & Boundary concentration \\
\hline $\mathrm{NH}_{3}$ & $\mathrm{C}_{1}$ & $\rho_{\mathrm{w}}$ & $7.6 \mathrm{mgN} / \mathrm{kg}$ & $7.6 \mathrm{mgN} / \mathrm{kg}$ \\
\hline $\mathrm{NH}_{3(\mathrm{~b})}$ & $\mathrm{C}_{2}$ & $\mathrm{Ph}_{\mathrm{b}} \rho_{\mathrm{wb}} \theta_{\mathrm{b}} / \mathrm{A}$ & $7.6 \mathrm{mgN} / \mathrm{kg}$ & - \\
\hline $\mathrm{NO}_{3}$ & $\mathrm{C}_{3}$ & $\rho_{\mathrm{w}}$ & $0.35 \mathrm{mgN} / \mathrm{kg}$ & $0.35 \mathrm{mgN} / \mathrm{kg}$ \\
\hline $\mathrm{NO}_{3(\mathrm{~b})}$ & $\mathrm{C}_{4}$ & $\mathrm{Ph}_{b} \rho_{\mathrm{wb}} \theta_{\mathrm{b}} / \mathrm{A}$ & $0.35 \mathrm{mgN} / \mathrm{kg}$ & - \\
\hline $\mathrm{OPO}_{4}$ & $\mathrm{C}_{5}$ & $\rho_{w}$ & $0.4 \mathrm{mgP} / \mathrm{kg}$ & $0.4 \mathrm{mgP} / \mathrm{kg}$ \\
\hline $\mathrm{OPO}_{4(\mathrm{~b})}$ & $\mathrm{C}_{6}$ & $\mathrm{Ph}_{\mathrm{b}} \rho_{\mathrm{wb}} \theta_{\mathrm{b}} / \mathrm{A}$ & $0.4 \mathrm{mgP} / \mathrm{kg}$ & - \\
\hline PHYT & $\mathrm{C}_{7}$ & $\rho_{\mathrm{w}}$ & $6.0 \mathrm{mgC} / \mathrm{kg}$ & $6.0 \mathrm{mgC} / \mathrm{kg}$ \\
\hline $\mathrm{PHYT}_{(\mathrm{b})}$ & $\mathrm{C}_{8}$ & $\mathrm{Ph}_{\mathrm{b}} \rho_{\mathrm{wb}} \theta_{\mathrm{b}} / \mathrm{A}$ & $6.0 \mathrm{mgC} / \mathrm{kg}$ & - \\
\hline $\mathrm{CH}_{2} \mathrm{O}$ & $\mathrm{C}_{9}$ & $\rho_{\mathrm{w}}$ & $5.25 \mathrm{mgO}_{2} / \mathrm{kg}$ & $5.25 \mathrm{mgO}_{2} / \mathrm{kg}$ \\
\hline $\mathrm{CH}_{2} \mathrm{O}_{(\mathrm{p})}$ & $\mathrm{C}_{10}$ & SS & $0.15 \mathrm{mgO}_{2} / \mathrm{mg}$ & $0.15 \mathrm{mgO}_{2} / \mathrm{mg}$ \\
\hline $\mathrm{CH}_{2} \mathrm{O}_{(\mathrm{b})}$ & $\mathrm{C}_{11}$ & $\mathrm{Ph}_{\mathrm{b}} \rho_{\mathrm{wb}} \theta_{\mathrm{b}} / \mathrm{A}$ & $5.25 \mathrm{mgO}_{2} / \mathrm{kg}$ & - \\
\hline $\mathrm{CH}_{2} \mathrm{O}_{(\mathrm{bp})}$ & $\mathrm{C}_{12}$ & $\mathrm{PBS} / \mathrm{A}$ & $0.0136 \mathrm{mgO}_{2} / \mathrm{mg}$ & - \\
\hline $\mathrm{O}_{2}$ & $\mathrm{C}_{13}$ & $\rho_{\mathrm{w}}$ & $3.6 \mathrm{mgO}_{2} / \mathrm{kg}$ & $3.6 \mathrm{mgO}_{2} / \mathrm{kg}$ \\
\hline $\mathrm{O}_{2(\mathrm{~b})}$ & $\mathrm{C}_{14}$ & $\mathrm{Ph}_{b} \rho_{\mathrm{wb}} \theta_{\mathrm{b}} / \mathrm{A}$ & $3.6 \mathrm{mgO}_{2} / \mathrm{kg}$ & - \\
\hline ON & $\mathrm{C}_{15}$ & $\rho_{w}$ & $1.15 \mathrm{mgN} / \mathrm{kg}$ & $1.15 \mathrm{mgN} / \mathrm{kg}$ \\
\hline $\mathrm{ON}_{(\mathrm{p})}$ & $\mathrm{C}_{16}$ & SS & $0.0 \mathrm{mgN} / \mathrm{mg}$ & $0 \mathrm{mgN} / \mathrm{mg}$ \\
\hline $\mathrm{ON}_{(\mathrm{b})}$ & $\mathrm{C}_{17}$ & $\mathrm{Ph}_{b} \rho_{\mathrm{wb}} \theta_{\mathrm{b}} / \mathrm{A}$ & $1.15 \mathrm{mgN} / \mathrm{kg}$ & - \\
\hline $\mathrm{ON}_{(\mathrm{bp})}$ & $\mathrm{C}_{18}$ & $\mathrm{PBS} / \mathrm{A}$ & $0.0 \mathrm{mgN} / \mathrm{mg}$ & - \\
\hline $\mathrm{OP}$ & $\mathrm{C}_{19}$ & $\rho_{\mathrm{w}}$ & $0.28 \mathrm{mgP} / \mathrm{kg}$ & $0.28 \mathrm{mgP} / \mathrm{kg}$ \\
\hline $\mathrm{OP}_{(\mathrm{p})}$ & $\mathrm{C}_{20}$ & SS & $0.00343 \mathrm{mg} \mathrm{P} / \mathrm{mg}$ & $0.00343 \mathrm{mgP} / \mathrm{mg}$ \\
\hline $\mathrm{OP}_{(\mathrm{b})}$ & $\mathrm{C}_{21}$ & $\mathrm{Ph}_{b} \rho_{\mathrm{wb}} \theta_{\mathrm{b}} / \mathrm{A}$ & $0.28 \mathrm{mgP} / \mathrm{kg}$ & - \\
\hline $\mathrm{OP}_{(\mathrm{bp})}$ & $\mathrm{C}_{22}$ & $\mathrm{PBS} / \mathrm{A}$ & $0.00031 \mathrm{mgP} / \mathrm{mg}$ & - \\
\hline
\end{tabular}


considered. Once the resulting 8 equations are solved for $\mathrm{C}_{1}$ through $\mathrm{C}_{8}$, Eq. (13) is used to calculate the dynamics of $\mathrm{O}_{2(\mathrm{~g})}$, and Eqs. (9) through (12) are used to calculate the amount of $\mathrm{H}^{+}, \mathrm{H}_{2} \mathrm{O}, \mathrm{CO}_{2}$, and $\mathrm{N}_{2}$ that must be supplied to conserve mass for protons, water, carbon dioxide, and nitrogen. In an open aqueous system, the amount of nitrogen in the atmosphere can be considered infinite. Also in a large water body, the amount of water needed to maintain mass conservation due to biogeochemical processes can be easily met. A more troublesome issue is what will be the source of protons $\mathrm{H}^{+}$and carbon dioxide $\mathrm{CO}_{2}$ to maintain their conservation with respect to reactions. In general, the mass of $\mathrm{CO}_{2}$ in the atmosphere is large compared to mass of carbonate in the river system. For any system, if this nagging question cannot be clearly answered, then the $\mathrm{pH}$ and partial pressure of $\mathrm{CO}_{2}$ would probably be important factors controlling reaction rates and inducing additional biogeochemical processes. Under such circumstances, one probably has to revisit the rate equations and conduct research to uncover additional reactions relevant to the system under investigation.

The diagonalization approach allows one to formulate some of the rate equations one by one. For example, the reaction rate $\mathrm{R}_{11}$ can be calculated by plotting the concentration of $E_{1}$ versus time in which $E_{1}$ is the linear combination of $\mathrm{C}_{1}, \mathrm{C}_{2}, \mathrm{C}_{4}, \mathrm{C}_{5}, \mathrm{C}_{6}, \mathrm{C}_{7}$, and $\left[\mathrm{O}_{2(\mathrm{~g})}\right.$ ] [see Eq. (1) in Table 5].

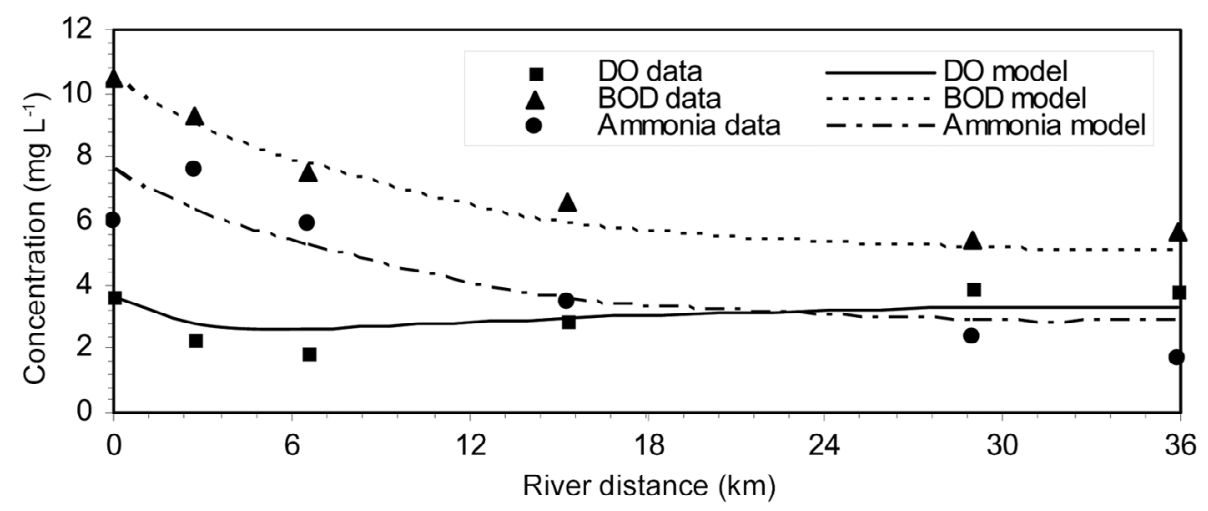

Fig. 4. Measured and simulated chemical concentrations in the Des Moines River.

Table 4. Simplified EUTRO5 reaction network.

\begin{tabular}{|c|c|c|c|}
\hline No. & Mechanism & Reaction & Rate \\
\hline 1 & PHYT growth & $\mathrm{a}_{\mathrm{nc}} \mathrm{NH}_{3}+\mathrm{a}_{\mathrm{pc}} \mathrm{OPO}_{4}+\mathrm{CO}_{2}+\mathrm{H}_{2} \mathrm{O} \rightarrow \mathrm{PHYT}+\frac{32}{12} \cdot \mathrm{O}_{2}$ & $\mathrm{R}_{1}$ \\
\hline 2 & PHYT growth related nitrate reduction & $\mathrm{a}_{\mathrm{nc}} \mathrm{NO}_{3} \rightarrow \mathrm{a}_{\mathrm{nc}} \mathrm{NH}_{3}+\frac{48}{12} \cdot \mathrm{O}_{2}$ & $\mathrm{R}_{2}$ \\
\hline 3 & PHYT death-endogenous respiration & $\mathrm{PHYT}+\frac{32}{12} \cdot \mathrm{O}_{2} \rightarrow \mathrm{CO}_{2}+\mathrm{H}_{2} \mathrm{O}+\mathrm{a}_{\mathrm{nc}} \mathrm{ON}+\mathrm{a}_{\mathrm{pc}} \mathrm{OP}$ & $\mathrm{R}_{3}$ \\
\hline 4 & PHYT death-parasitization & $\mathrm{PHYT} \rightarrow \mathrm{a}_{\mathrm{oc}} \mathrm{CH}_{2} \mathrm{O}+\mathrm{a}_{\mathrm{nc}} \mathrm{ON}+\mathrm{a}_{\mathrm{pc}} \mathrm{OP}$ & $\mathrm{R}_{4}$ \\
\hline 5 & PHYT death-herbivorous grazing & $\mathrm{PHYT} \rightarrow \mathrm{a}_{\mathrm{oc}} \mathrm{CH}_{2} \mathrm{O}+\mathrm{a}_{\mathrm{nc}} \mathrm{ON}+\mathrm{a}_{\mathrm{pc}} \mathrm{OP}$ & $\mathrm{R}_{5}$ \\
\hline 6 & PHYT death-promoted oxidation of ON & $\mathrm{a}_{\mathrm{nc}} \mathrm{ON} \rightarrow \mathrm{a}_{\mathrm{nc}} \mathrm{NH}_{3}$ & $\mathrm{R}_{6}$ \\
\hline 7 & PHYT death-promoted oxidation of OP & $\mathrm{a}_{\mathrm{pc}} \mathrm{OP} \rightarrow \mathrm{a}_{\mathrm{pc}} \mathrm{OPO}_{4}$ & $\mathrm{R}_{7}$ \\
\hline 8 & Re-aeration & $\mathrm{O}_{2(\mathrm{~g})} \rightarrow \mathrm{O}_{2}$ & $\mathrm{R}_{8}$ \\
\hline 9 & Carbonaceous oxidation & $\mathrm{CH}_{2} \mathrm{O}+\mathrm{O}_{2} \rightarrow \mathrm{CO}_{2}+\mathrm{H}_{2} \mathrm{O}$ & $\mathrm{R}_{9}$ \\
\hline 10 & Nitrogen mineralization & $\mathrm{ON} \rightarrow \mathrm{NH}_{3}$ & $\mathrm{R}_{10}$ \\
\hline 11 & Nitrification & $\mathrm{NH}_{3}+\frac{64}{14} \cdot \mathrm{O}_{2} \rightarrow \mathrm{NO}_{3}^{-}+\mathrm{H}_{2} \mathrm{O}+\mathrm{H}^{+}$ & $\mathrm{R}_{11}$ \\
\hline 12 & De-nitrification & $1.25 \mathrm{CH}_{2} \mathrm{O}+\frac{14}{32} \cdot \mathrm{NO}_{3}+\mathrm{H}^{+} \rightarrow 1.25 \mathrm{CO}_{2}+0.5 \mathrm{~N}_{2}+1.75 \mathrm{H}_{2} \mathrm{O}$ & $\mathrm{R}_{12}$ \\
\hline 13 & Phosphorous mineralization & $\mathrm{OP} \rightarrow \mathrm{OPO}_{4}$ & $\mathrm{R}_{13}$ \\
\hline
\end{tabular}


Table 5. Working equations for the reaction-based diagonalization approach.

\begin{tabular}{|c|c|}
\hline No. & Working Equations \\
\hline 1 & $\frac{\mathrm{dE}}{\mathrm{dt}}=\frac{\mathrm{d}\left[\frac{23}{4} \cdot \frac{32}{14} \mathrm{C}_{1}-\left(\frac{3}{2} \cdot \frac{32}{12} / \mathrm{a}_{\mathrm{nc}}-\frac{23}{4} \cdot \frac{32}{14}\right) \mathrm{C}_{2}+\left(\frac{32}{12}+\mathrm{a}_{\mathrm{nc}} \frac{23}{4} \cdot \frac{32}{14}\right) \mathrm{C}_{4}+\mathrm{C}_{5}-\mathrm{C}_{6}+\frac{23}{4} \cdot \frac{32}{14} \mathrm{C}_{7}-\mathrm{O}_{2(8)}\right]}{\mathrm{dt}}=-5 \cdot \frac{32}{14} \mathrm{R}_{11}$ \\
\hline 2 & $\frac{d E_{2}}{d t}=\frac{d\left(C_{2}+C_{1}+a_{n e} C_{4}+C_{7}\right)}{d t}=-\frac{14}{32} R_{12}$ \\
\hline 3 & $\frac{\mathrm{dE}_{3}}{\mathrm{dt}}=\frac{\mathrm{dC}_{3}}{\mathrm{dt}}=-\mathrm{a}_{\mathrm{pc}} \mathrm{R}_{1}+\mathrm{a}_{\mathrm{pc}} \mathrm{R}_{7}+\mathrm{R}_{13}$ \\
\hline 4 & $\frac{d E_{4}}{d t}=\frac{d\left[\left(1-a_{n c} \frac{5}{4} \cdot \frac{12}{14}\right) C_{4}-\frac{5}{4} \cdot \frac{12}{14} C_{1}-\frac{5}{4} \cdot \frac{12}{14} C_{2}+40 C_{3}+\frac{12}{32} C_{5}-\frac{5}{4} \cdot \frac{12}{14} C_{7}\right]}{d t}=-R_{3}+40 a_{p c} R_{7}-R_{9}+40 R_{13}$ \\
\hline 5 & $\frac{d E_{5}}{d t}=\frac{d\left(C_{5}-\frac{5}{4} \cdot \frac{32}{14} C_{1}-\frac{5}{4} \cdot \frac{32}{14} C_{2}-a_{n c} \frac{5}{4} \cdot \frac{32}{14} C_{4}-\frac{5}{4} \cdot \frac{32}{14} C_{7}\right)}{d t}=\frac{32}{12} R_{4}+\frac{32}{12} R_{5}-R_{9}$ \\
\hline 6 & 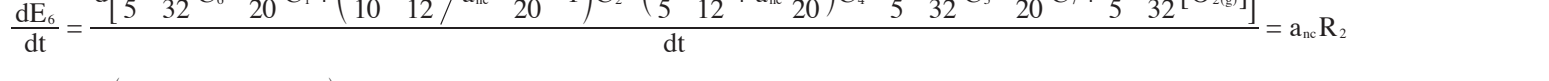 \\
\hline 7 & $\frac{d E_{7}}{d t}=\frac{d\left(\frac{a_{p c}}{a_{n c}} C_{7}+C_{3}+a_{p c} C_{4}\right)}{d t}=-a_{p c} R_{6}+a_{p c} R_{7}-\frac{a_{p c}}{a_{n c}} R_{10}+R_{13}$ \\
\hline $8 \mathrm{OP}$ & $\frac{\mathrm{d} \mathrm{T}_{1}}{\mathrm{dt}}=\frac{\mathrm{d}\left(\mathrm{C}_{8}+\mathrm{C}_{3}+\mathrm{a}_{\mathrm{pc}} \mathrm{C}_{4}\right)}{\mathrm{dt}}=0$ \\
\hline $9 \mathrm{H}^{+}$ & $\frac{\mathrm{dT} \mathrm{T}_{2}}{\mathrm{dt}}=\frac{\mathrm{d}\left[\left[\mathrm{H}^{+}\right]+\frac{3}{20} \cdot \frac{1}{14} \mathrm{C}_{1}-\left(\frac{3}{10} \cdot \frac{1}{12} / \mathrm{a}_{\mathrm{nc}}-\frac{3}{20} \cdot \frac{1}{14}\right) \mathrm{C}_{2}+\left(\frac{1}{5} \cdot \frac{1}{12}+\mathrm{a}_{\mathrm{nc}} \frac{3}{20} \cdot \frac{1}{14}\right) \mathrm{C}_{4}+\frac{1}{5} \cdot \frac{1}{32} \mathrm{C}_{5}-\frac{1}{5} \cdot \frac{1}{32} \mathrm{C}_{6}+\frac{3}{20} \cdot \frac{1}{14} \mathrm{C}_{7}-\frac{1}{5} \cdot \frac{1}{32}\left[\mathrm{O}_{2(\mathrm{~s})}\right]\right]}{\mathrm{dt}}=($ \\
\hline $10 \mathrm{H}_{2} \mathrm{O}$ & $\frac{\mathrm{dT} \mathrm{T}_{3}}{\mathrm{dt}}=\frac{\mathrm{d}\left[\left[\mathrm{H}_{2} \mathrm{O}\right]+\frac{33}{20} \cdot \frac{18}{14} \mathrm{C}_{1}+\left(\frac{33}{20} \cdot \frac{18}{14}-\frac{3}{10 \mathrm{a}_{\mathrm{nc}}} \cdot \frac{18}{12}\right) \mathrm{C}_{2}+\left(\frac{6}{5} \cdot \frac{18}{12}+\mathrm{a}_{\mathrm{nc}} \frac{33}{20} \cdot \frac{18}{14}\right) \mathrm{C}_{4}+\frac{6}{5} \cdot \frac{18}{32} \mathrm{C}_{5}-\frac{1}{5} \cdot \frac{18}{32} \mathrm{C}_{6}+\frac{33}{20} \cdot \frac{18}{14} \mathrm{C}_{7}-\frac{1}{5} \cdot \frac{18}{32}\left[\mathrm{O}_{2(8)}\right]\right]}{\mathrm{dt}}=$ \\
\hline $11 \mathrm{CO}_{2}$ & $\frac{\mathrm{dT}_{4}}{\mathrm{dt}}=\frac{\mathrm{d}\left(\left[\mathrm{CO}_{2}\right]+\mathrm{C}_{4}+\frac{12}{32} \mathrm{C}_{5}\right)}{\mathrm{dt}}=0$ \\
\hline $12 \mathrm{~N}_{2}$ & $\frac{\mathrm{d} \mathrm{T}_{5}}{\mathrm{dt}}=\frac{\mathrm{d}\left(\left[\mathrm{N}_{2}\right]+\mathrm{C}_{1}+\mathrm{C}_{2}+\mathrm{a}_{\mathrm{ne}} \mathrm{C}_{4}+\mathrm{C}_{7}\right)}{\mathrm{dt}}=0$ \\
\hline $13 \mathrm{O}_{2(\mathrm{~g})}$ & $\frac{\mathrm{dE}}{\mathrm{dt}}=\frac{\mathrm{d}\left[\mathrm{O}_{2(\mathrm{~g})}\right]}{\mathrm{dt}}=-\mathrm{R}_{8}$ \\
\hline
\end{tabular}

Similarly, reaction rates $\mathrm{R}_{12}, \mathrm{R}_{2}$, and $\mathrm{R}_{8}$ can be calculated from the dynamics of $E_{2}, E_{6}$, and $E_{8}$, respectively. Because linearly dependent reactions are present in the system, one cannot formulate all rate equations independently. To do so, one has to design an experimental system in which only linearly independent reactions occur in order to individually and mechanistically formulate rate equations.

\section{EXAMPLE PROBLEM}

This hypothetical example was designed to demonstrate the capability of the model to simulate coupled sediment and reactive chemical transport with all ten types of reactions presented in Fig. 1.

A $20 \mathrm{~km}$-long stream reach with a width of $20 \mathrm{~m}$ and a water depth of $2 \mathrm{~m}$ was considered. The domain was dis- cretized into 100 equal size elements (200 m each). The stream velocity was constant at $1 \mathrm{~m} \mathrm{~s}^{-1}$. Only one size of cohesive sediment was taken into account with a settling speed of $1.0 \times 10^{-6} \mathrm{~m} \mathrm{~s}^{-1}$, erodibility of $0.15 \mathrm{~g} \mathrm{~m}^{-2} \mathrm{~s}^{-1}$, critical shear stress for deposition of $2.85 \mathrm{~g} \mathrm{~m}^{-1} \mathrm{~s}^{-2}$, and critical shear stress for erosion of $2.48 \mathrm{~g} \mathrm{~m}^{-1} \mathrm{~s}^{-2}$. Manning's roughness was assumed to equal 0.02 .

Three chemicals were considered which are dissolved in the mobile water phase (CMW1, CMW2, and CMW3), in the immobile water phase (CIMW1, CIMW2, and CIMW3), in suspended sediment phase (CS1, CS2, and CS3), and in bed sediment phase (CB1, CB2, and CB3). Additionally, one suspended precipitate (SP3) and one bed precipitate (BP3) were modeled yielding a total of 14 water quality constituents. These constituents were subject to the interactions summarized in Table 6 . Totally, there are twenty reactions, 
Table 6. Chemical reactions considered in the example problem.

\begin{tabular}{|c|c|c|}
\hline Reaction and rate parameter & Type * & No. \\
\hline CMW1 + CMW2 $\leftrightarrow$ CMW3 $\left(\mathrm{k}_{\mathrm{eq}}=0.4 \mathrm{~m}^{3} / \mathrm{g}\right)$ & 1 & $\mathrm{R}_{1}$ \\
\hline $\begin{array}{l}\mathrm{CMW} 1+\mathrm{SS} \leftrightarrow \mathrm{CS} 1+\mathrm{SS} \\
\mathrm{CMW} 2+\mathrm{SS} \leftrightarrow \mathrm{CS} 2+\mathrm{SS} \\
\mathrm{CMW} 3+\mathrm{SS} \leftrightarrow \mathrm{CS} 3+\mathrm{SS} \\
\left(\mathrm{k}_{\mathrm{f}}=0.001 \mathrm{~m}^{3} / \mathrm{gSS} / \mathrm{s}, \mathrm{k}_{\mathrm{b}}=0.0 \mathrm{~s}^{-1}\right)\end{array}$ & 2 & $\begin{array}{l}\mathrm{R}_{2} \\
\mathrm{R}_{3} \\
\mathrm{R}_{4}\end{array}$ \\
\hline $\begin{array}{l}\mathrm{CMW} 1+\mathrm{BS} \leftrightarrow \mathrm{CB} 1+\mathrm{BS} \\
\mathrm{CMW} 2+\mathrm{BS} \leftrightarrow \mathrm{CB} 2+\mathrm{BS} \\
\mathrm{CMW} 3+\mathrm{BS} \leftrightarrow \mathrm{CB} 3+\mathrm{BS} \\
\left(\mathrm{k}_{\mathrm{f}}=0.00001 \mathrm{~m}^{2} / \mathrm{gBS} / \mathrm{s}, \mathrm{k}_{\mathrm{b}}=0.0 \mathrm{P} / \mathrm{A} \mathrm{m}^{-1} \mathrm{~s}^{-1}\right)\end{array}$ & 4 & $\begin{array}{l}\mathrm{R}_{5} \\
\mathrm{R}_{6} \\
\mathrm{R}_{7}\end{array}$ \\
\hline $\begin{array}{l}\mathrm{CS} 1 \leftrightarrow \mathrm{CB} 1\left(\mathrm{k}_{\mathrm{f}}=\text { Depo }_{1} \mathrm{P} / \mathrm{AgSS} / \mathrm{m}^{3} / \mathrm{s}, \mathrm{k}_{\mathrm{b}}=\operatorname{Eros}_{1} \mathrm{P} / \mathrm{AgBS} / \mathrm{m}^{3} / \mathrm{s}\right) \\
\mathrm{CS} 2 \leftrightarrow \mathrm{CB} 2\left(\mathrm{k}_{\mathrm{f}}=\operatorname{Depo}_{2} \mathrm{P} / \mathrm{AgSS}^{3} / \mathrm{m}^{3} / \mathrm{s}, \mathrm{k}_{\mathrm{b}}=\operatorname{Eros}_{2} \mathrm{P} / \mathrm{AgBS} / \mathrm{m}^{3} / \mathrm{s}\right) \\
\mathrm{CS} 3 \leftrightarrow \mathrm{CB} 3\left(\mathrm{k}_{\mathrm{f}}=\operatorname{Depo}_{3} \mathrm{P} / \mathrm{AgSS}^{3} / \mathrm{m}^{3} / \mathrm{s}, \mathrm{k}_{\mathrm{b}}=\operatorname{Eros}_{3} \mathrm{P} / \mathrm{AgS} / \mathrm{m}^{3} / \mathrm{s}\right)\end{array}$ & 10 & $\begin{array}{l}\mathrm{R}_{8} \\
\mathrm{R}_{9} \\
\mathrm{R}_{10}\end{array}$ \\
\hline $\begin{array}{l}\text { CMW1 } \leftrightarrow \text { CIMW1 } \\
\text { CMW2 } \leftrightarrow \text { CIMW2 } \\
\text { CMW3 } \leftrightarrow \text { CIMW3 } \\
\left(\mathrm{k}_{\mathrm{f}}=0.0001 \mathrm{~s}^{-1}, \mathrm{k}_{\mathrm{b}}=0.0 \mathrm{Ph}_{\mathrm{b}} \theta_{\mathrm{b}} / \mathrm{A} \mathrm{s}^{-1}\right)\end{array}$ & 9 & $\begin{array}{l}\mathrm{R}_{11} \\
\mathrm{R}_{12} \\
\mathrm{R}_{13}\end{array}$ \\
\hline $\begin{array}{l}\text { CIMW1 + CIMW2 } \leftrightarrow \text { CIMW3 } \\
\left(\mathrm{k}_{\mathrm{f}}=0.0002 \mathrm{Ph}_{\mathrm{b}} \theta_{\mathrm{b}} / \mathrm{A} \mathrm{m}^{3} / \mathrm{g} / \mathrm{s}, \mathrm{k}_{\mathrm{b}}=0.0005 \mathrm{Ph}_{\mathrm{b}} \theta_{\mathrm{b}} / \mathrm{A} \mathrm{s}^{-1}\right)\end{array}$ & 5 & $\mathrm{R}_{14}$ \\
\hline $\begin{array}{l}\mathrm{CIMW} 1+\mathrm{BS} \leftrightarrow \mathrm{CB} 1+\mathrm{BS} \\
\mathrm{CIMW} 2+\mathrm{BS} \leftrightarrow \mathrm{CB} 2+\mathrm{BS} \\
\mathrm{CIMW} 3+\mathrm{BS} \leftrightarrow \mathrm{CB} 3+\mathrm{BS} \\
\left(\mathrm{k}_{\mathrm{f}}=0.00001 \mathrm{Ph}_{\mathrm{b}} \theta_{\mathrm{b}} / \mathrm{A} \mathrm{m}^{2} / \mathrm{gBS} / \mathrm{s}, \mathrm{k}_{\mathrm{b}}=0.0 \mathrm{P} / \mathrm{A} \mathrm{m}^{-1} \mathrm{~s}^{-1}\right)\end{array}$ & 6 & $\begin{array}{l}\mathrm{R}_{15} \\
\mathrm{R}_{16} \\
\mathrm{R}_{17}\end{array}$ \\
\hline $\begin{array}{l}\text { CMW2 } \leftrightarrow \mathrm{P} \\
\left(\mathrm{k}_{\mathrm{f}}=0.0002 \mathrm{~s}^{-1}, \mathrm{k}_{\mathrm{b}}=0.02 \mathrm{~g} / \mathrm{m}^{3} / \mathrm{ATM} / \mathrm{s}, \mathrm{P}=0.0025 \mathrm{ATM}\right)\end{array}$ & 8 & $\mathrm{R}_{18}$ \\
\hline $\begin{array}{l}\mathrm{CMW} 3 \leftrightarrow \mathrm{SP} 3 \\
\left(\mathrm{k}_{\mathrm{f}}=0.001 \mathrm{~s}^{-1}, \mathrm{k}_{\mathrm{b}}=0.000001 \mathrm{~s}^{-1}\right)\end{array}$ & 3 & $\mathrm{R}_{19}$ \\
\hline $\begin{array}{l}\text { CIMW3 } \leftrightarrow \text { BP } 3 \\
\left(\mathrm{k}_{\mathrm{f}}=0.0001 \mathrm{Ph}_{\mathrm{b}} \theta_{\mathrm{b}} / \mathrm{A} \mathrm{s}^{-1}, \mathrm{k}_{\mathrm{b}}=0.0000001 \mathrm{Ph}_{\mathrm{b}} \theta_{\mathrm{b}} / \mathrm{A} \mathrm{s}^{-1}\right)\end{array}$ & 7 & $\mathrm{R}_{20}$ \\
\hline
\end{tabular}

among which, $R_{1}$ is an equilibrium aqueous complexation reaction among the three dissolved chemicals in the mobile water phase; $R_{2}$ through $R_{4}$ are kinetic adsorption reactions of the three dissolved chemicals in the mobile water phase onto the suspended sediment phase; $\mathrm{R}_{5}$ through $\mathrm{R}_{7}$ are kinetic adsorption reactions of the three dissolved chemicals in the mobile water phase onto the bed sediment phase; $R_{8}$ through $\mathrm{R}_{10}$ are kinetic sedimentation reactions of the three particulates between the suspended and bed sediment phases; $R_{11}$ through $R_{13}$ describe diffusive mass transfer of the three dissolved chemicals between the mobile and immobile water phases; $R_{14}$ is a kinetic aqueous complexation reaction among the three dissolved chemicals in the immobile water phase; $R_{15}$ through $R_{17}$ are kinetic adsorption reactions of the three dissolved chemicals in the immobile water phase onto the bed sediment phase; $R_{18}$ is a kinetic volatilization reaction of the second dissolved chemical in the mobile water phase; $\mathbf{R}_{19}$ is a kinetic precipitation/dissolution reaction between the third dissolved chemical in the mobile water phase and suspended precipitate; and $\mathrm{R}_{20}$ is a kinetic precipitation/dissolution reaction between the third dissolved chemical in the immobile water phase and bed precipitate.

The model for this example involves 1 equilibrium and 19 kinetic reactions and yields 13 transport equations for non-equilibrium variables and 1 equilibrium equations following incomplete decomposition, which are solved to obtain the 14 water quality constituents (Table 7 ). In the table, $\rho_{\mathrm{i}}$, the density of the phase associated with water quality constituent $\mathrm{i}$, is $\rho_{\mathrm{w}}$ for CMW1-CMW3 and SP3; SS for CS1-CS3; $\mathrm{Ph}_{\mathrm{b}} \rho_{\mathrm{wb}} \theta_{\mathrm{b}} / \mathrm{A}$, for CIMW1-CIMW3 and BP3; and $\mathrm{P} \cdot \mathrm{BS} / \mathrm{A}$, for $\mathrm{CB} 1-\mathrm{CB} 3$, where the column water density $\rho_{\mathrm{w}}=1.0 \mathrm{~kg} \mathrm{~L}^{-1}$, bed water density $\rho_{\mathrm{wb}}=1.0 \mathrm{~kg} \mathrm{~L}^{-1}$, river 
Table 7. Equations obtained through decomposition in the example problem.

\begin{tabular}{|c|c|}
\hline Equations & Type* \\
\hline$\frac{\partial\left(\mathrm{AE}_{1}\right)}{\partial \mathrm{t}}+\mathrm{L}\left(\mathrm{E}_{1}^{\mathrm{m}}\right)=\mathrm{A}\left(-\mathrm{R}_{2}-\mathrm{R}_{4}-\mathrm{R}_{5}-\mathrm{R}_{7}-\mathrm{R}_{11}-\mathrm{R}_{13}-\mathrm{R}_{19}\right)$ where $\mathrm{E}_{1}=\mathrm{E}_{1}^{\mathrm{m}}=\rho_{\mathrm{CMW} 1} \mathrm{C}_{\mathrm{CMW} 1}+\rho_{\mathrm{CMW}} \mathrm{C}_{\mathrm{CMW} 3}$ & 1 \\
\hline$\frac{\partial\left(\mathrm{AE}_{2}\right)}{\partial \mathrm{t}}+\mathrm{L}\left(\mathrm{E}_{2}^{\mathrm{m}}\right)=\mathrm{A}\left(-\mathrm{R}_{3}-\mathrm{R}_{4}-\mathrm{R}_{6}-\mathrm{R}_{7}-\mathrm{R}_{12}-\mathrm{R}_{13}-\mathrm{R}_{18}-\mathrm{R}_{19}\right)$ where $\mathrm{E}_{2}=\mathrm{E}_{2}^{\mathrm{m}}=\rho_{\mathrm{CMW} 2} \mathrm{C}_{\mathrm{CMW} 2}+\rho_{\mathrm{CMW} 3} \mathrm{C}_{\mathrm{CMW} 3}$ & 1 \\
\hline$\frac{\partial\left(\mathrm{AE}_{3}\right)}{\partial \mathrm{t}}+\mathrm{L}\left(\mathrm{E}_{3}^{\mathrm{m}}\right)=\mathrm{A}\left(\mathrm{R}_{2}-\mathrm{R}_{8}\right)$ where $\mathrm{E}_{3}=\mathrm{E}_{3}^{\mathrm{m}}=\rho_{\mathrm{CS} 1} \mathrm{C}_{\mathrm{CS} 1}$ & 1 \\
\hline$\frac{\partial\left(\mathrm{AE}_{4}\right)}{\partial \mathrm{t}}+\mathrm{L}\left(\mathrm{E}_{4}^{\mathrm{m}}\right)=\mathrm{A}\left(\mathrm{R}_{3}-\mathrm{R}_{9}\right)$ where $\mathrm{E}_{4}=\mathrm{E}_{4}^{\mathrm{m}}=\rho_{\mathrm{CS} 2} \mathrm{C}_{\mathrm{CS} 2}$ & 1 \\
\hline$\frac{\partial\left(\mathrm{AE}_{5}\right)}{\partial \mathrm{t}}+\mathrm{L}\left(\mathrm{E}_{5}^{\mathrm{m}}\right)=\mathrm{A}\left(\mathrm{R}_{4}-\mathrm{R}_{10}\right)$ where $\mathrm{E}_{5}=\mathrm{E}_{5}^{\mathrm{m}}=\rho_{\mathrm{cs} 3} \mathrm{C}_{\mathrm{cS} 3}$ & 1 \\
\hline$\frac{\partial\left(\mathrm{AE}_{6}\right)}{\partial \mathrm{t}}+\mathrm{L}\left(\mathrm{E}_{6}^{\mathrm{m}}\right)=\mathrm{A}\left(\mathrm{R}_{5}+\mathrm{R}_{8}+\mathrm{R}_{15}\right)$ where $\mathrm{E}_{6}=\rho_{\mathrm{CB} 1} \mathrm{C}_{\mathrm{CB} 1}$ and $\mathrm{E}_{6}^{\mathrm{m}}=0$ & 1 \\
\hline$\frac{\partial\left(\mathrm{AE}_{7}\right)}{\partial \mathrm{t}}+\mathrm{L}\left(\mathrm{E}_{7}^{\mathrm{m}}\right)=\mathrm{A}\left(\mathrm{R}_{6}+\mathrm{R}_{9}+\mathrm{R}_{16}\right)$ where $\mathrm{E}_{7}=\rho_{\mathrm{CB} 2} \mathrm{C}_{\mathrm{CB} 2}$ and $\mathrm{E}_{7}^{\mathrm{m}}=0$ & 1 \\
\hline$\frac{\partial\left(\mathrm{AE}_{8}\right)}{\partial \mathrm{t}}+\mathrm{L}\left(\mathrm{E}_{8}^{\mathrm{m}}\right)=\mathrm{A}\left(\mathrm{R}_{7}+\mathrm{R}_{10}+\mathrm{R}_{17}\right)$ where $\mathrm{E}_{8}=\rho_{\mathrm{CB} 3} \mathrm{C}_{\mathrm{CB} 3}$ and $\mathrm{E}_{8}^{\mathrm{m}}=0$ & 1 \\
\hline$\frac{\partial\left(\mathrm{AE}_{9}\right)}{\partial \mathrm{t}}+\mathrm{L}\left(\mathrm{E}_{9}^{\mathrm{m}}\right)=\mathrm{A}\left(\mathrm{R}_{11}-\mathrm{R}_{14}-\mathrm{R}_{15}\right)$ where $\mathrm{E}_{9}=\rho_{\mathrm{CIMw} 1} \mathrm{C}_{\mathrm{CIMw} 1}$ and $\mathrm{E}_{9}^{\mathrm{m}}=0$ & 1 \\
\hline$\frac{\partial\left(\mathrm{AE}_{10}\right)}{\partial \mathrm{t}}+\mathrm{L}\left(\mathrm{E}_{10}^{\mathrm{m}}\right)=\mathrm{A}\left(\mathrm{R}_{12}-\mathrm{R}_{14}-\mathrm{R}_{16}\right)$ where $\mathrm{E}_{10}=\rho_{\mathrm{CIMW} 2} \mathrm{C}_{\mathrm{CIMW} 2}$ and $\mathrm{E}_{10}^{\mathrm{m}}=0$ & 1 \\
\hline$\frac{\partial\left(\mathrm{AE}_{11}\right)}{\partial \mathrm{t}}+\mathrm{L}\left(\mathrm{E}_{11}^{\mathrm{m}}\right)=\mathrm{A}\left(\mathrm{R}_{13}+\mathrm{R}_{14}-\mathrm{R}_{17}-\mathrm{R}_{20}\right)$ where $\mathrm{E}_{11}=\rho_{\mathrm{CIMW} 3} \mathrm{C}_{\mathrm{CIMw} 3}$ and $\mathrm{E}_{11}^{\mathrm{m}}=0$ & 1 \\
\hline$\frac{\partial\left(\mathrm{AE}_{12}\right)}{\partial \mathrm{t}}+\mathrm{L}\left(\mathrm{E}_{12}^{\mathrm{m}}\right)=\mathrm{AR}_{19}$ where $\mathrm{E}_{12}=\mathrm{E}_{12}^{\mathrm{m}}=\rho_{\mathrm{SP} 3} \mathrm{C}_{\mathrm{SP} 3}$ & 1 \\
\hline$\frac{\partial\left(\mathrm{AE}_{13}\right)}{\partial \mathrm{t}}+\mathrm{L}\left(\mathrm{E}_{13}^{\mathrm{m}}\right)=\mathrm{AR}_{20}$ where $\mathrm{E}_{13}=\rho_{\mathrm{BP} 3} \mathrm{C}_{\mathrm{BP} 3}$ and $\mathrm{E}_{13}^{\mathrm{m}}=0$ & 1 \\
\hline $\mathrm{C}_{\mathrm{CMW}_{3}}=0.4 \mathrm{C}_{\mathrm{CMW}_{1}} \mathrm{C}_{\mathrm{CMW}_{2}}$ & 2 \\
\hline
\end{tabular}

* Type 1 is a non-equilibrium variable transport equation and Type 2 is an equilibrium equation.

bed depth $\mathrm{h}_{\mathrm{b}}=0.1 \mathrm{~m}$, and bed sediment porosity $\theta_{\mathrm{b}}=0.5$. Among the 13 non-equilibrium-variables, the $6^{\text {th }}$ through $11^{\text {th }}$ and the $13^{\text {th }}$ contain no mobile constituents and are thus not solved in the advective-dispersive transport step. Therefore, instead of solving 7 advective-dispersive transport equations for mobile water quality constituents in a primitive approach, we need only to solve 6 advective-dispersive transport equations for non-equilibrium variables. Since the equilibrium reaction is decoupled and not included in the transport equations, the formulation is intrinsically parsimonious.

Initially, only sediment exists in the domain of interest with a suspended concentration SS of $1 \mathrm{~g} \mathrm{~m}^{-3}$ and a bed concentration BS of $50 \mathrm{~g} \mathrm{~m}^{-2}$. As the simulation starts, Dirichlet boundary conditions are applied at the upstream boundary where suspended sediment has a constant concentration of $1 \mathrm{~g} \mathrm{~m}^{-3}$, all three dissolved chemicals in the mobile water phase have constant concentrations of $1 \mathrm{mg} \mathrm{kg}^{-1}$, and all other mobile constituents have zero concentrations. A variable boundary condition is applied to the downstream boundary node. The longitudinal dispersivity is $80 \mathrm{~m}$. A 90000 -second simulation is performed with a fixed time-step size of 150 seconds.

Figure 5 shows a trend of increasing suspended sediment concentration with distance downstream, and decreas- ing bed sediment with time, indicating that deposition is less than erosion under the conditions in this example. The concentration of CMW1 also decreases with distance since it is subject to adsorption but desorption does not occur. In the zone near the Dirichlet boundary, the concentration distribution curve of CMW1 is not smooth. Due to equilibrium among the three dissolved chemicals in the mobile water phase, the concentration of CMW1 increases to its equilibrium value. The only source of dissolved chemicals in the immobile water phase is the corresponding dissolved chemicals in the mobile water phase. Therefore, the concentration distribution of CIMW1 shows the similar pattern of CMW1.

Since dissolved concentrations are low in the downstream region, most of the chemical transport occurs via suspended sediment. Because erosion is greater than deposition, we observe increases in CS1 with time and decreases in CB1 with distance. Since the particulate chemicals in the bed sediment phase result not only from dissolved chemicals in the mobile water phase, but also from those in the immobile water phase, the decrease of CB1 with distance also reflects the similar pattern of CMW1 and CIMW1.

Since the major source of suspended precipitate in the downstream region is transported from the upstream region, we observe an increase of suspended precipitate concentra- 

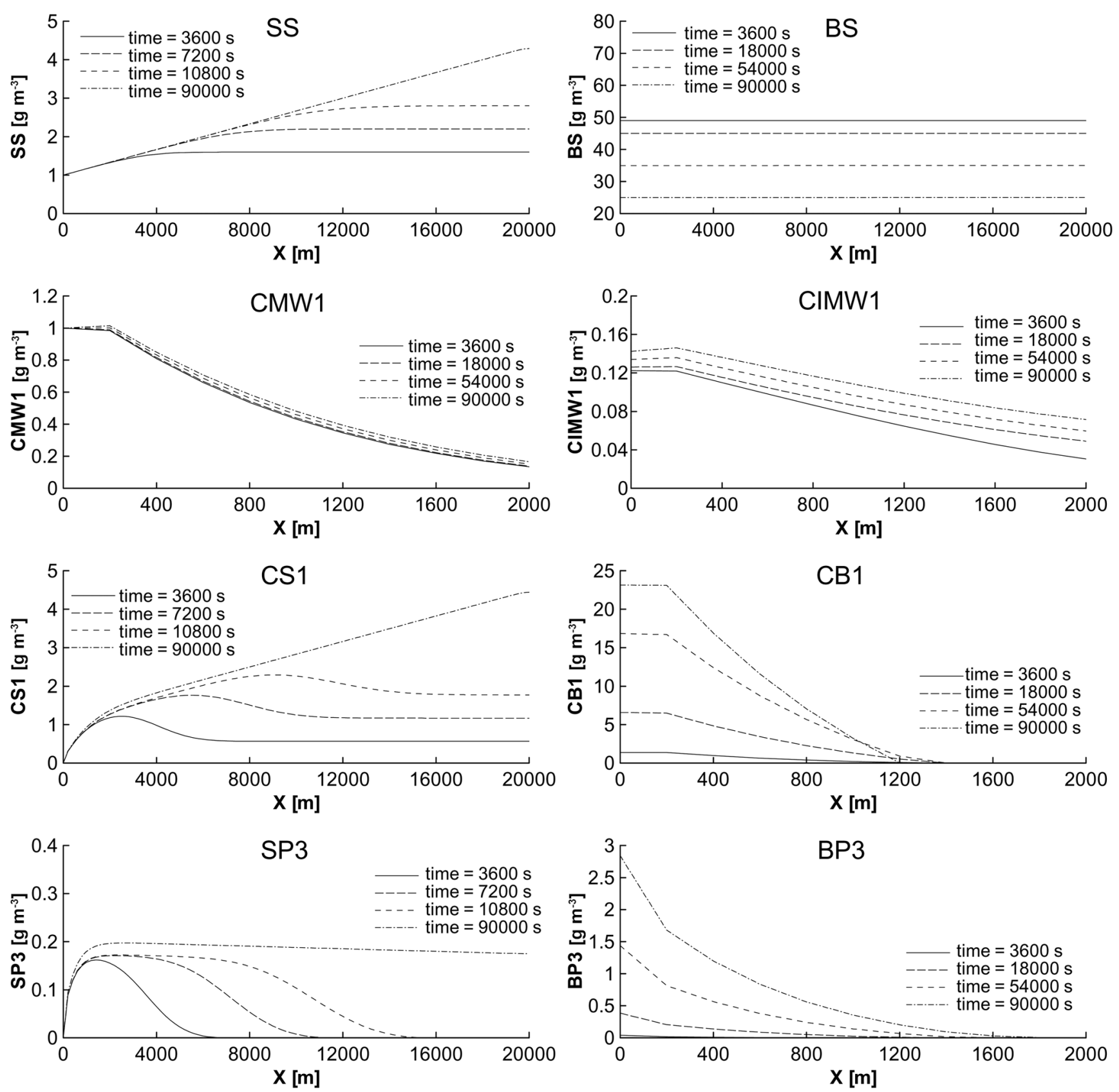

Fig. 5. Concentration profiles for the example problem simulating sediment and reactive chemical transport with ten types of reactions: (a) suspended sediment SS; (b) bed sediment BS; (c) dissolved chemical in the mobile water phase CMW1; (d) dissolved chemical in the immobile water phase CIMW1; (e) particulate chemical in the suspended sediment phase CS1; (f) particulate chemical in the bed sediment phase CB1; (g) suspended precipitate SP3; and (h) bed precipitate BP3.

tion with time. Since the bed precipitate is involved in the precipitation reaction only, its concentration decreases with distance, reflecting the decrease in dissolved chemical concentration in the immobile phase.

\section{CONCLUSIONS AND REMARKS}

This paper presents the matrix decomposition approach for a reaction-based water quality model. The fate and transport of water quality constituents was mathematically described by a system of advective-dispersive-reactive transport equations with user-prescribed kinetic and/or equilibrium reactions. Through incomplete decomposition of the system of water quality constituent transport equations via Gauss-Jordan column reduction of the reaction network by pivoting on equilibrium reactions, equilibrium and kinetic reactions are decoupled, which may reduce the number of partial differential advective-dispersive transport equations 
and enables more robust numerical integration. Through complete matrix decomposition by further pivoting on linear independent kinetic reactions, some rate equations are allowed to be formulated individually and conservation of component species is explicitly enforced when the component transport equations are solved. The general paradigm of modeling water quality transport presented here can simulate any reaction network describing conceptualized biogeochemical processes. For examples, the biogeochemical processes embodied in the widely used WASP5, QUAL2E, and CE-QUAL-ICM have been successfully transformed and casted in reaction networks (Yeh et al. 2001), implying that these three models can be simulated using the code developed here. The model has been applied to and validated with a field problem using the eutrophication model of WASP5. The capability of the model to simulate general problems beyond WASP5, QUAL2E, and CE-QUAL-ICM has been demonstrated using two examples with complex mixed equilibrium and kinetic reactions. The challenge of applying the present model to any practical field problem is the transformation of biogeochemical processes into reaction networks. The challenge is by no means easy, and it usually requires years of hard core basic research. The model provides a protocol for modeling various conceptualizations to undertake such endeavors.

\section{NOTATION}

$\mathbf{0}_{\mathbf{i}} \quad$ zero submatrix of reduced $\boldsymbol{v}, \mathbf{i}=1,2,3,4$;

A stream cross-sectional area $\left[\mathrm{L}^{2}\right]$;

BS bed sediment concentration $\left[\mathrm{M} \mathrm{L}^{-3}\right]$;

C water quality constituent concentration vector incorporating $\rho_{\mathrm{i}}$;

$\mathbf{C}_{\mathrm{A}}$ water quality constituent concentration vector incorporating $\rho_{\mathrm{i}}$ multiplied by A;

$\mathrm{C}_{\mathrm{i}}$ concentration of water quality constituent i $\left[\mathrm{M} \mathrm{M}^{-1}\right]$;

$\mathbf{D}_{\mathbf{i}} \quad$ "diagonal" submatrix of reduced $\boldsymbol{v}, \mathbf{i}=1,2$;

$\mathrm{E}_{\mathrm{i}} \quad$ concentration of the $\mathrm{i}$-th reaction-extent $\left[\mathrm{M} \mathrm{L}^{-3}\right]$;

$\mathrm{E}_{\mathrm{i}}^{\mathrm{m}} \quad$ concentration of mobile part of the i-th reaction-extent $\left[\mathrm{M} \mathrm{L}^{-3}\right]$;

$E_{i}^{i m} \quad$ concentration of immobile part of the $i$-th reactionextent $\left[\mathrm{M} \mathrm{L}^{-3}\right]$;

$\mathrm{h}_{\mathrm{b}} \quad$ stream bed depth [L];

L transport operator incorporating source terms;

M total number of water quality constituents;

$M_{i m}$ number of immobile water quality constituents;

$\mathrm{M}_{\mathrm{m}} \quad$ number of mobile water quality constituents;

NR total number of reactions;

$\mathrm{N}_{\mathrm{C}} \quad$ number of components;
$\mathrm{N}_{\mathrm{DK}}$ number of linearly dependent kinetic reactions;

$\mathrm{N}_{\mathrm{E}}$ number of equilibrium reactions;

$\mathrm{N}_{\mathrm{IK}}$ number of linearly independent kinetic reactions;

p time step number;

P stream cross-sectional wetted perimeter [L];

$\mathbf{R}$ reaction rate vector with NR reaction rates as its components;

$\mathbf{r}_{\mathrm{DK}}$ linear dependent kinetic reaction rate subvector of $\mathbf{r}$ with size of $\mathrm{N}_{\mathrm{DK}}$;

$\mathbf{r}_{\mathrm{E}}$ equilibrium reaction rate subvector of $\mathbf{r}$ with size of $\mathrm{N}_{\mathrm{E}}$;

$\mathrm{R}_{\mathrm{i}}$ production rate of reaction-extent $\mathrm{i}$ due to biogeochemical reactions $\left[\mathrm{M} \mathrm{L}^{-3} \mathrm{~T}^{-1}\right]$;

$\mathbf{r}_{\mathrm{IK}}$ linear independent kinetic reaction rate subvector of $\mathbf{r}$ with size of $\mathrm{N}_{\mathrm{IK}}$;

$\left.r_{i}\right|_{N R}$ production rate of water quality constituent $i$ due to all NR reactions $\left[\mathrm{M} \mathrm{L}^{-3} \mathrm{~T}^{-1}\right]$;

$r_{k} \quad$ rate of the $k$-th reaction $\left[\mathrm{M} \mathrm{L}^{-3} \mathrm{~T}^{-1}\right]$;

$\mathrm{r}_{\mathrm{k}}^{\mathrm{b}} \quad$ backward rate of reaction $\mathrm{k}\left[\mathrm{M} \mathrm{L}^{-3} \mathrm{~T}^{-1}\right]$;

$\mathrm{r}_{\mathrm{k}}^{\mathrm{f}} \quad$ forward rate of reaction $\mathrm{k}\left[\mathrm{M} \mathrm{L}^{-3} \mathrm{~T}^{-1}\right]$;

SS suspended sediment concentration $\left[\mathrm{M} \mathrm{L}^{-3}\right]$;

$\mathrm{t}$ time [T];

$\mathbf{U}$ unit matrix;

$\mathbf{U}^{\prime}$ reduced $\mathbf{U}$ matrix through completele decomposition;

A diagonal matrix with $\alpha_{\mathrm{i}}$ as its diagonal component;

$\alpha_{i} \quad$ mobility of water quality constituent $i$;

$\boldsymbol{\alpha}^{\prime}$ reduced $\boldsymbol{\alpha}$ matrix through completele decomposition;

$\theta_{\mathrm{b}} \quad$ porosity of the bed sediment $\left[\mathrm{L}^{3} \mathrm{~L}^{-3}\right]$;

$\mu_{\mathrm{ik}}$ reactant reaction stoichiometry of $\mathrm{i}$-th constituent in k-th reaction;

$\boldsymbol{v}_{\mathrm{i}} \quad$ nonzero submatrix of reduced $\boldsymbol{v}, \mathbf{i}=1,2,3$;

$\nu_{\mathrm{ik}} \quad$ product reaction stoichiometry of i-th constituent in k-th reaction;

$\rho_{\mathrm{i}} \quad$ density of the phase associated with water quality constituent i $\left[\mathrm{M} \mathrm{L}^{-3}\right]$;

$\rho_{\mathrm{w}} \quad$ density of column water [ $\left[\mathrm{M} \mathrm{L}^{-3}\right]$; and

$\rho_{\mathrm{wb}} \quad$ density of bed water $\left[\mathrm{M} \mathrm{L}^{-3}\right]$.

Acknowledgements This research is funded by the Ministry of Science and Technology of the People's Republic of China under Grant \# 2010CB951702 and US EPA-Science To Achieve Results Program under Grant \# R-82795602 with University of Central Florida. Although the research described in this article has been supported by the US Environmental Protection Agency, this article has not been 
subjected to the Agency's peer and administrative review and therefore does not necessarily reflect the view of the Agency and no official endorsement should be inferred. The research was also partially funded by the US Department of Energy, Office of Science, Biological and Environmental Research Programs. Oak Ridge National Laboratory is managed by UT-Battelle LLC for the DOE under contract DE-AC05-00OR22725. Fan Zhang was partially supported by the Chinese Academy of Sciences Hundred Talent Program during the preparation of the manuscript. Gour-Tsyh Yeh was partially supported by Preparatory Office, Taiwan National Center for Typhoon and Flood Research during the preparation of the manuscript.

\section{REFERENCES}

Ambrose, R. B., T. A. Wool, and J. L. Martin, 1993: The Water Quality Analysis Simulation Program, WASP5 Part A: Model Documentation. USEPA Environmental Research Laboratory, Athens, GA.

Bierman, V. J. Jr. and R. T. James, 1995: A preliminary modeling analysis of water quality in Lake Okeechobee, Florida: Diagnostic and sensitivity analyses. Water Res., 29, 2767-2775, doi: 10.1016/0043-1354(95)00 117-4. [Link]

Brown, L. C. and T. O. Barnwell Jr., 1987: The Enhanced Stream Water Quality Models QUAL2E and QUAL2EUNCAS: Documentation and User Manual. Environmental Research Laboratory, Office of Research and Development, USEPA, Athens, GA 30613.

Cerco, C. F. and T. Cole, 1995: User's Guide to the CEQUAL-ICM Three-Dimensional Eutrophication Model. Release Version 1.0, US Army Engineer Waterways Experiment Station, Vicksburg, MS.

Cheng, H. P., G. T. Yeh, and J. R. Cheng, 2000: A numerical model simulating reactive transport in shallow water domains: Model development and demonstrative applications. Adv. Environ. Res., 4, 187-209, doi: 10.1016/ S1093-0191(00)00015-0. [Link]

Chilakapati, A., 1995: RAFT: A Simulator for ReActive Flow and Transport of Groundwater Contaminants. PNL-10636, Pacific Northwest National Laboratory, Richland, WA.

DiToro, D. M., 1976: Combining chemical equilibrium and phytoplankton models - A general methodology. In: Canale, R. P. (Author), Modeling Biochemical Processes in Aquatic Ecosystems, Ann Arbor Science Press, $398 \mathrm{pp}$.

Fang, Y., G. T. Yeh, and W. D. Burgos, 2003: A general paradigm to model reaction-based biogeochemical processes in batch systems. Water Resour. Res., 39, 1083-1118, doi: 10.1029/2002WR001694. [Link]

Fang, Y., S. B. Yabusaki, and G. T. Yeh, 2006: A General Simulator for Reaction-Based Biogeochemical
Processes. Comput. Geosci., 32, 64-72, doi: 10.1016/j. cageo.2005.05.003. [Link]

Gu, R. and M. Dong, 1998: Water quality modeling in the watershed-based approach for waste load allocations. Water Sci.Technol., 38, 165-172, doi: 10.1016/S02731223(98)00746-X. [Link]

Lung, W. S. and C. E. Larson, 1995: Water quality modeling of upper Mississippi River and Lake Pepin. J. Environ. Eng., 121, 691-699, doi: 10.1061/(ASCE)07339372(1995)121:10(691). [Link]

Mann, U., 2000: New design formulation of chemical reactors with multiple reactions: I. Basic concepts. Chem. Eng. Sci., 55, 991-1008, doi: 10.1016/S0009-2509(99) 00364-4. [Link]

SomlyóDy, L., M. Henze, L. Koncsos, W. Rauch, P. Reichert, P. Shanahan, and P. Vanrolleghem, 1998: River water quality modelling: III. Future of the art. Water Sci. Technol., 38, 253-260, doi: 10.1016/S0273-1223 (98)00662-3. [Link]

Steefel, C. I. and V. P. Cappellen, 1998: Preface: Reactive transport modeling of natural systems. J. Hydrol., 209, $1-7$.

Stumm, W. and J. J. Morgan, 1981: Aquatic Chemistry: An Introduction Emphasizing Chemical Equilibria in Natural Waters. Environmental Science and Technology: A Wiley-Interscience Series of Texts and Monographs, John Wiley \& Sons, $780 \mathrm{pp}$.

Tufford, D. L. and H. N. McKellar, 1999: Spatial and temporal hydrodynamic and water quality modeling analysis of a large reservoir on the South Carolina (USA) coastal plain. Ecol.Model., 114, 137-173, doi: 10.1016/ S0304-3800(98)00122-7. [Link]

Yeh, G. T. and V. S. Tripathi, 1989: A critical evaluation of recent developments in hydrogeochemical transport models of reactive multichemical components. Water Resour. Res., 25, 93-108, doi: 10.1029/WR025i001p 00093. [Link]

Yeh, G. T. and F. Zhang, 2005: A General Paradigm of Modeling One-dimensional River/Stream Watershed Water Quality. Department of Civil and Environmental Engineering, University of Central Florida, Orlando, Florida, USA.

Yeh, G. T., H. P. Cheng, J. R. Cheng, and J. H. Lin, 1998: A numerical model simulating water flow and contaminant and sediment transport in watershed systems of 1-D stream-river network, 2-D overland regime, and 3-D subsurface media (WASH123D: Version 1.0). Waterway Experiment Station, US Army Corps of Engineering, Vicksburg, MS 39180-6199.

Yeh, G. T., W. D. Burgos, and J. M. Zachara, 2001: Modeling and measuring biogeochemical reactions: system consistency, data needs, and rate formulations. Adv. Environ. Res., 5, 219-237, doi: 10.1016/S1093-0191 (00)00057-5. [Link] 
Zhang,F., G. T. Yeh, J.C.Parker, and P. M. Jardine, 2008: A reaction-based river/stream water quality model: Model development and numerical schemes. J. Hydrol., 348, 496-509, doi: 10.1016/j.jhydrol.2007.10.020. [Link]
Zheng, L., C. Chen, and F. Y. Zhang, 2004: Development of water quality model in the Satilla River Estuary, Georgia. Ecol. Model., 178, 457-482, doi: 10.1016/j. ecolmodel.2004.01.016. [Link] 